\title{
Systematic Screening Strategies for Identifying Elite Plant Growth Promoting Rhizobacteria for Coconut (Cocos nucifera L.)
}

\author{
Priya George ${ }^{1,2^{*}}$, Alka Gupta ${ }^{1}$, Murali Gopal ${ }^{1}$, Litty Thomas ${ }^{1}$ and George V. Thomas ${ }^{1,3}$ \\ ${ }^{1}$ Microbiology Section, ICAR-Central Plantation Crops Research Institute, Kudlu P.O., \\ Kasaragod - 671 124, India \\ ${ }^{2}$ ICAR-National Institute of Abiotic stress Management, Baramati, Pune-413115, India \\ ${ }^{3}$ Council for Food Research and Development, Konni, Kerala, India
}

*Corresponding author

\section{A B S T R A C T}

Elite strains of plant growth promoting rhizobacteria were proficiently selected based on the strategic screening approaches. These included in vitro testing for plant growth promotion attributes, seedling bioassay under controlled and under greenhouse conditions.

\section{Keywords}

Screening PGPR,

Fluorescent

pseudomonads, Bacillus

spp., Coconut, Plant

growth promoting traits

Article Info

Accepted:

10 April 2018

Available Online:

10 May 2018
A total of 483 morphologically distinct bacteria [156 rhizosphere fluorescent Pseudomonas spp. (RSF), 206 rhizosphere Bacillus spp. (RSB) and 121 endophytic Bacillus spp. (EB)] were isolated from the roots and rhizosphere soil of coconut palms growing in different states of India. These were characterized for various plant growth promotion traits that have direct and indirect effects on plant growth. All the isolates were assessed and given scores based on their in vitro assay of plant growth promotion traits. A total of 129 isolates, which secured more than 11 points, were further selected for seedling bioassay under controlled conditions. Greenhouse trial was conducted with 72 isolates and results further proved the efficiency of screened isolates for plant growth promotion. This strategic screening helped in shortlisting a total of 20 best PGPR ( 7 fluorescent Pseudomonas spp. and 13 Bacillus spp.) out of 483 isolates. The present study clearly showed that the rhizosphere of coconut palms harbour varied populations of fluorescent pseudomonads and Bacillus spp. that possess diverse plant growth promotion properties, and some of the efficient ones could be developed in to bioinoculants for use in coconut nurseries and plantations.

\section{Introduction}

The coconut is a tropical tree species, mainly grown and harvested by small-scale farmers. Production of coconuts is concentrated on the island and coastal areas, with Indonesia, Philippines, India and Brazil being the major producers. India is the third largest coconut producing country in the world, its cultivation spread in 1.89 million hectares of land (FAOSTAT data, 2016). Coconut is classified as a "functional food" because it provides many health benefits beyond its nutritional content. As every bit of the coconut is used for human intake, its cultivation practices should be in more healthy, organic and eco-friendly manner. Hence, it is desirable that the natural wonder 'coconut' is not exposed to harmful 
and hazardous synthetic chemicals as fertilizers and pesticides. Research on the microorganisms in the root region has proven beneficial to the eco-friendly farming practices. Rhizosphere and endorhizosphere microorganisms have a great impact on root biology, influence nutrition, plant growth, uptake and development (Mantelin and Touraine, 2004). Rhizosphere organisms that have been well studied for their beneficial effects on plant growth and health are the nitrogen-fixing bacteria, mycorrhizal fungi, plant growth-promoting rhizobacteria (PGPR), biocontrol microorganisms, mycoparasitic fungi, and protozoa (Mendes et al., 2013). PGPR were first used for agricultural purposes in the former Soviet Union and India in the early $20^{\text {th }}$ century and are now being tested for plant growth promotion worldwide (Lucy et $a l ., 2004)$ in many plants including many crop plants (Dutta and Thakur, 2017; Vargas et al., 2017). The most common non-symbiotic genera with plant growth promotion activity included Pseudomonas, Bacillus, Azospirillum, Azotobacter, Enterobacter and Serratia (Geroge et al., 2013; Vargas et al., 2017; Dutta and Thakur, 2017). As a perennial crop, coconut palm is likely to recruit highly potent 'candidates' from the pool of soil microorganisms to maintain a 'rhizomicrobiome' for enduring mutual interaction between them. The occurrence of higher population of beneficial plant-associated microbes was reported in the rhizosphere of coconut palms (Gopal et al., 2005; Rajendran et al., 2007; George et al., 2013). Enhanced plant growth and nutrient uptake has been reported in seedlings of perennial crops such as cocoa (Thomas et al., 2011), oil palm (Amir et al., 2005) and oak (Domenech et al., 2004) upon inoculation with PGPR. Among rhizosphere microorganisms, the fluorescent pseudomonads have emerged as the largest and potentially most promising group of cultivable PGPR with the production of versatile metabolites (Santoro et al., 2016).
Likewise, Bacillus spp. attracted considerable attention because of their advantages over other PGPR strains in inoculant formulations, stable maintenance in rhizosphere soil, and greater potential in sustainable agriculture (Govindasamy et al., 2010).

An earlier report on the ability of PGPR such as Bacillus coagulans and Brevibacillus brevis improving the seedling growth of coconut (Gupta et al., 2006) forms the basis for this initiative on a very detailed and meticulous study of PGPR in coconut. Towards this aim, a study was taken up involving collection of soil and root samples from the rhizosphere of coconut palms growing in different locations in five southern states of India. A total of 483 morphologically distinct fluorescent Pseudomonas spp. and Bacillus spp. were isolated and purified from these samples. We affirmed the antagonistic activity and phosphate solubilization associated with some of these isolates in our previous reports (George et al., 2011, 2012a, b). Here we propose a screening strategy to select potent plant growth promoting rhizobacteria (PGPR) with multiple PGP traits from this microbial collection that could be developed into bioinoculants to enhance the health, vigour and growth of coconut seedlings.

\section{Materials and Methods}

\section{Isolation of fluorescent Pseudomonas spp. and Bacillus spp.}

The root and rhizospheric soil samples collected from yielding, healthy coconut palms growing in Kerala, Karnataka, Tamil Nadu, Andhra Pradesh and Maharashtra states of India (Fig. 1a) were used for isolation of fluorescent Pseudomonas spp. and Bacillus spp. The enumeration studies in coconut palms clearly indicated that the fluorescent pseudomonads and Bacillus spp. occurred in good numbers in the rhizosphere of this 
perennial plantation crop (George et al., 2011, 2012b; Fig. 1b, c). A total of 156 rhizospheric fluorescent Pseudomonas spp. (RSF), 206 rhizospheric Bacillus spp. (RSB) and 121 root endophytic Bacillus spp. (EB) isolates, representing each location were purified on King's B agar (KBA), Gould's S2 agar and Nutrient agar (NA). They were identified as fluorescent Pseudomonas spp. and Bacillus spp. based on the preliminary identification methods. The methods for collection, population, isolation and preliminary identification of the isolates were done as reported elsewhere (George et al., 2011, 2012b).

\section{Screening of isolates in vitro for plant growth promotion traits}

All the isolates were characterized for their direct and indirect plant growth promoting traits such as growth on $\mathrm{N}$-free medium (Jensen's medium, Himedia), phosphate solubilization (Pikovskaya's agar, Himedia) (George et al., 2012b), production of IAA (Brick et al., 1991), 1-aminocyclopropane carboxylic acid (ACC)-deaminase activity (Klee et al., 1991), production of siderophores (Schwyn and Neilands, 1987), ammonification (Cappuccino and Sherman, 1992), production of HCN (Bakker and Schippers, 1987) and chitinase activity (Renwick et al., 1991). The ability of isolates to produce antibiotics was detected by agar well technique (Fuhrmann, 1994). Antifungal activity was detected against two different coconut pathogens (Thielaviopsis paradoxa and Ganoderma applanatum) (George et al., 2012b). For studying these properties, bacterial suspension having $10^{8}$ c.f.u. $\mathrm{ml}^{-1}$ was spot inoculated or streaked on different media as per the requirement of the procedure. All the isolates were assessed and scored in a scale of 1 to 3 based on their performance in above plant growth promotion traits (Table 1). Maximum 3 points were given to $\mathrm{HCN}$ production, growth on $\mathrm{N}$-free medium, high ammonifiers and good growth on minimal media containing ACC. Antibiotic producers were assessed based on the inhibition zone size, siderophore producers according to the diameter of orange halo and phosphate solubilizers and chitin degradation based on the size of clearing zones on a scale of 1 to 3 . Antifungal activity was evaluated according to their percentage of inhibition.

\section{Screening of isolates by seedling bioassay in plant growth chamber}

Selected isolates were grown in King's B (KB)/Nutrient Broth (NB) for $24 \mathrm{~h}$ at $30{ }^{\circ} \mathrm{C}$ in a refrigerated incubator shaker (Innova Model 4335, USA). Paddy seeds (Oryza sativa cv. Aiswarya) were surface sterilized with $0.1 \%$ $\mathrm{HgCl}_{2}$ for 4 minutes and washed repeatedly with sterile distilled water. Seeds were immersed in bacterial culture broth $\left(10^{8}\right.$ cells $\mathrm{ml}^{-1}$ ) for 10 minutes and then transferred to the petriplates (5 seeds per plate) containing soft water agar $(0.7 \%)$ and incubated in an Environmental Growth Chamber (Sanyo, Japan) at $25^{\circ} \mathrm{C}, 85 \% \mathrm{RH}, 10 \mathrm{~h}$ light and $14 \mathrm{~h}$ dark cycle for 10 days. Three replications were maintained. Control was maintained by placing surface-sterilized seeds (without culture) for germination and growth under same conditions. Shoot and root lengths of the seedlings were measured. Data were statistically analysed using Duncan's multiple range test.

\section{Screening of isolates by seedling bioassay in greenhouse}

Surface sterilized paddy seeds were sown in $11 \times 9 \mathrm{~cm}$ sized plastic cups (four seeds/cup) filled with unsterile soil: sand mixture $(\mathrm{pH}$ 5.3 , EC $90 \mu \mathrm{S} / \mathrm{cm}$ ) in 3:1 ratio. One $\mathrm{ml}$ of $24 \mathrm{~h}$ old bacterial culture $\left(10^{8}\right.$ cells $\left.\mathrm{ml}^{-1}\right)$ was applied on each seed. The cups were placed in greenhouse (Average Temp: $27{ }^{\circ} \mathrm{C}$, Average 
Humidity: $91 \%$ ). After germination, seedlings were thinned to 2 numbers per cup. Ten replications were maintained for each microorganism. Control treatment received surface-sterilized seeds without application of bacterial culture. Seedlings were uprooted after 20 days of sowing and their shoot and root lengths and fresh and dry weights were recorded. Data were statistically analysed using Duncan's multiple range test. Percentage of increase in total seedling length, fresh weight, and dry weight were calculated. The populations of rhizospheric fluorescent Pseudomonas spp./Bacillus spp. were enumerated in the rhizosphere soil of paddy seedlings by serial dilution and pour plating method on S2/NA media, respectively.

\section{Identification of screened isolates}

The screened plant growth promoting rhizobacteria (PGPR) were identified based on 16S rRNA gene sequence analysis. Genomic DNA of the PGPR was isolated by GenElute Bacterial Genomic DNA Kit (Sigma, USA) and fragment of $16 \mathrm{~S}$ rDNA gene was amplified by PCR from the isolated genomic DNA using the universal primers F27/R1492 as described earlier (George et al., 2013).

\section{Statistical analysis}

The data were analyzed using analysis of variance (ANOVA) and the means were compared using Duncan's Multiple Range Test (DMRT) at $\mathrm{P}=0.05$ level using version 15 of SPSS.

\section{Results and Discussion}

\section{In vitro characterization for plant growth promotion traits}

The overall results showed that all the isolates had strong tendencies for one or more of the plant growth promoting (PGP) traits tested and none were negative for all traits. The percent of RSF and bacilli showing plant growth promoting traits is depicted in Figure 2a. Results indicated that ammonifying bacteria were preponderant PGPR in the rhizosphere and roots of coconut. Other traits like $\mathrm{HCN}$ production and antibiotic production were found in a few isolates. Nitrogen fixing ability, chitinase activity and antagonism were found more in rhizospheric bacilli, whereas IAA production, phosphate solubilization and siderophore were found more in RSF.

A total of 217 isolates (48 RSB, $37 \mathrm{~EB}, 132$ RSF), whose colonies were immobilized on Whatman filter paper and then treated with Salkowski reagent, produced pink colour on the filter paper (Fig. 2b). Thirty two fluorescent Pseudomonas spp. and 17 Bacillus spp. exhibited high colour intensity indicative of higher levels of IAA production. Eighty percent of all isolates showed growth on minimal medium containing ACC as the sole source of nitrogen as it would seem to possess low to high activity of ACC deaminase enzyme. A total of 84 isolates showed luxuriant growth on $\mathrm{N}$-free medium. This included 21 bacilli from the rhizosphere, 60 bacilli from endorhizosphere and 3 fluorescent pseudomonads from the rhizosphere. Almost half of the endophytic bacilli showed growth on $\mathrm{N}$-free medium and were presumptive nitrogen fixers. In the present study, most of the fluorescent pseudomonads (147 nos.) were found to be phosphate solubilizers. Two Pseudomonas spp. KnSF208 and KnSF227 produced maximum clearing zone of $42 \mathrm{~mm}$ diameter (Fig. 2c). The siderophore positive isolates produced orange halo surrounding their colonies on CAS agar medium (Fig. 2d). Again, fluorescent Pseudomonas spp. KiSF17 and KiSF33 showed highest affinity for $\mathrm{Fe}$ sequestration as indicated by orange halo (30 $\mathrm{mm}$ dia.). The production of $\mathrm{HCN}$ could be seen only in $4 \%$ of the isolates which included 11 Bacillus spp. and 8 fluorescent 
Pseudomonas spp. and was indicated by change in colour of the filter paper from yellow to orange/brown (Fig. 2e). This study also revealed that chitinase activity was mostly limited to Bacillus spp. (131 isolates). Only one isolate of Pseudomonas sp. KiSF 13 produced clearing zone of $12 \mathrm{~mm}$ diameter on chitin medium (Fig. 2f). Antibiotic production was exhibited by 16 (10\% of the total) isolates (Fig. 2g). Growth of Ganoderma applanatum and Thielaviopsis paradoxa were inhibited by more than $90 \%$ isolates with percentage inhibition ranging from 44 to $91 \%$ on KBA (Fig. 3 a, b, c, d) / NA (Fig. 3 e, f, g, h) medium. Maximum antagonism that could be achieved by fluorescent Pseudomonas spp. and Bacillus spp. were $76 \%$ and $93 \%$, respectively, in terms of mycelial growth reduction.

All the isolates were assigned scores based on their performance in plant growth promoting traits. As equal importance were given to all the plant growth promoting traits tested, maximum three points were possible for each of the 11 parameters totaling 33 points (Table 1 ). A total of 129 isolates that scored $\geq 11$, were selected for further studies (Table 2). Among RSF, Pseudomonas sp. KiSF16 got maximum score of 21 points followed by Pseudomonas spp. PoSF 314 and KiSF17 with 19 points. Among Bacillus spp., Bacillus sp. PoEB5 isolated from the roots of coconut from Pollachi District of Tamil Nadu scored maximum (17) followed by Bacillus spp. RSB14 and KiEB25 (score of 16), isolated from coconut growing in Ratnagiri (Maharashtra) and Kidu (Karnataka), respectively.

\section{Effect of bacterial inoculation on paddy seedlings under controlled conditions}

Out of 21 RSF isolates, 13 isolates increased the length of paddy seedlings (ranging from 5 to $48 \%$ ) as compared to uninoculated control
(Fig. 4a) when tested under controlled temperature, humidity and light conditions. Maximum increase in total length was shown by paddy seedlings inoculated with Pseudomonas sp. ChSF180 from Chengannur, Kerala (48\%), followed by Pseudomonas sp. KiSF27 from Kidu, Karnataka (33\%) after 10 days growth period and were significantly different from uninoculated control in DMRT analysis. Nineteen out of 64 RSB positively influenced the growth of paddy seedlings under environmental growth chamber conditions. They showed 3-29\% increase in total length of paddy seedlings over respective uninoculated controls with maximum showed by the seedlings inoculated with Bacillus sp. ChSB2. Inoculation of paddy seeds with 22 EB isolates increased the total seedling length (upto $47 \%$ over control); many isolates increased either root length or shoot length. Bacillus sp. KiEB31 (47\% increase) performed better than other isolates in stimulating plant growth, which was also statistically significant over the other treatments. The bacterial isolates which showed plant growth promotion either in shoot or root were also selected for further studies. Hence, the initial collection of 483 isolates was shortlisted to 72 PGPR (12 RSF, 35 RSB and $25 \mathrm{~EB})$ after two screening approaches. The individual results of bacterial inoculation studies under controlled conditions are given in supplementary data (Table S1, S2 and S3) and summary of the results is depicted in Table 3.

Effect of bacterial inoculation on paddy seedlings under greenhouse conditions

In the 21-days-duration study with 72 isolates (12 RSF, 35 RSB and $25 \mathrm{~EB}$ ) conducted under greenhouse conditions, $10 \mathrm{RSF}$ isolates (out of 12 tested) increased all the growth parameters of paddy seedlings (Fig. 4b) with maximum increase recorded in Pseudomonas sp. KiSF27 $(36 \%)$ and two isolates increased at least one 
growth parameter. More than $100 \%$ increase in dry weight was observed amongst 8 RSF with Pseudomonas sp. KiSF24 (isolated from Kidu) recording $200 \%$ increase in dry weigh. Most of the growth parameters were found to be significantly different from the control.

Among 35 RSB tested, 10 isolates increased all parameters of paddy seedlings while others increased any one of the growth parameters. Bacillus sp. TSB16 isolated from Tumkur, Karnataka showed maximum increase in dry weight (163\%) and maximum increase in seedling length (48\%) was showed by Bacillus sp. ESB13 isolated from the rhizosphere of coconut growing in Ernakulam, Kerala. Six of the $25 \mathrm{~EB}$ increased all the growth parameters of paddy seedlings, while the remaining ones increased any one of the growth parameters. The maximum increase in total seedling length was shown by Bacillus sp. TEB4 (35\%), isolated from the roots of coconut palm growing in Tumkur, Karnataka.

Analysis of the rhizosphere soil of paddy seedlings showed the population of fluorescent pseudomonads to be higher when inoculated with RSF, compared to uninoculated control, after 20 days of planting.
It ranged from 3 to $5.92 \log _{10} \mathrm{cfu} \mathrm{g}^{-1}$ fresh weight of soil. Low population levels of fluorescent pseudomonads were detected in the non-treated control plants, up to $2 \log _{10} \mathrm{cfu}$ $\mathrm{ml}^{-1}$ fresh weight of soil. Population of Bacillus spp. in the rhizosphere region of paddy seedlings inoculated with RSB and EB ranged from $5.84-6.95 \log _{10} \mathrm{cfu} \mathrm{g}^{-1}$ of soil, when compared to control (average of 5.44 $\log _{10} \mathrm{~g}^{-1}$ of soil). The individual results of bacterial inoculation studies under greenhouse conditions are given in supplementary data (Table S4, S5 and S6).

\section{Selection and identification of PGPRs}

A total of 20 potential PGPR (7 RSF, 5 RSB and $8 \mathrm{~EB}$ ) were selected based on their overall performance in the above screening strategies. All of the selected PGPR had an assessment score $\geq 11$ points in functional traits and increased at least one of the growth parameter in plant assays.

Among the 13 Bacillus spp., 8 were root endophytes and rest were from the rhizosphere of coconut. The details of the isolates are given in Table 4 and their plant growth promoting traits in Table 5.

Table.1 Scheme for the assessment/ evaluation of PGPR

\begin{tabular}{|c|c|c|c|c|c|c|c|c|c|c|c|}
\hline \multirow[t]{2}{*}{ HCN } & \multirow{2}{*}{$\begin{array}{c}\text { PS } \\
(\text { Zone } \\
\text { in } \mathrm{mm})\end{array}$} & \multirow[t]{2}{*}{$\mathbf{N}$} & \multirow[t]{2}{*}{$\begin{array}{l}\text { Ammoni } \\
\text { fication* }\end{array}$} & \multirow{2}{*}{$\begin{array}{c}\text { Sideroph } \\
\text { ore (zone } \\
\text { in } \mathbf{m m} \text { ) }\end{array}$} & \multirow[t]{2}{*}{$\mathbf{I A A}$ * } & \multirow[t]{2}{*}{$\mathbf{A C C} *$} & \multirow{2}{*}{$\begin{array}{c}\text { Chitinase } \\
\text { (zone in } \\
\text { mm) }\end{array}$} & \multirow[t]{2}{*}{$\begin{array}{c}\text { Antibiotics } \\
\text { (zone in mm)* }\end{array}$} & \multicolumn{2}{|c|}{$\begin{array}{l}\text { Antago } \\
\text { nism*** }\end{array}$} & \multirow[t]{2}{*}{$\begin{array}{l}\text { Assessme } \\
\text { nt score } \neq\end{array}$} \\
\hline & & & & & & & & & $\overline{\text { GA }}$ & $\mathrm{TP}$ & \\
\hline & $1-14$ & & $\mathrm{~L}$ & $1-9$ & $\mathrm{~L}$ & $\mathrm{~L}$ & $1-8$ & $\mathrm{~L}$ & $\mathrm{~L}$ & $\mathrm{~L}$ & 1 \\
\hline & $15-28$ & & M & $10-19$ & M & M & $9-15$ & M & M & M & 2 \\
\hline+ & $\geq 29$ & + & $\mathrm{H}$ & $\geq 20$ & $\mathrm{H}$ & $\mathrm{H}$ & $\geq 16$ & $\mathrm{H}$ & $\mathrm{H}$ & $\mathrm{H}$ & 3 \\
\hline
\end{tabular}

HCN-Hydrogen cyanide production, PS- phosphate solubilization, N-growth on N-free medium, IAA- IAA production, ACC- ACC deaminase production, $\mathrm{mm}=$ diameter in $\mathrm{mm}$, GA- antagonism against Ganoderma applanatum, TP- antagonism against Thielaviopsis paradoxa," '+' = positive, ' - ' = negative, *The activity/production was qualitatively assessed in three broad categories: H (high), M- (medium), L (low), **Low $(\mathrm{L})$, medium $(\mathrm{M})$ and high $(\mathrm{H})$ represented inhibition ranges of 1-20, 21-40 and $\geq 41 \%$ and 40-59, 60-79 and 80$100 \%$ for Pseudomonas spp. and Bacillus spp., respectively. $\neq$ For negative reaction, no score was assigned. 
Table.2 Assessment of coconut RSF, RSB and EB based on plant growth promotion traits

\begin{tabular}{|l|c|c|c|c|c|c|}
\hline Group & $\begin{array}{c}\text { Number } \\
\text { of Isolates }\end{array}$ & \multicolumn{5}{|c|}{ Assessment points in range } \\
\hline RSF & 156 & $1-5$ & $\mathbf{6 - 1 0}$ & $\mathbf{1 1 - 1 5}$ & $\mathbf{1 6 - 2 0}$ & $\mathbf{2 1 - 2 5}$ \\
\hline RSB & 206 & 18 & 121 & 15 & 5 & 1 \\
\hline EB & 121 & 8 & 125 & 62 & 1 & - \\
Total & 483 & 40 & 314 & 119 & 9 & - \\
\hline
\end{tabular}

Total number of isolates selected for seedling 129 (RSF-21, RSB-63, EB-45) bioassay under controlled conditions

RSF - rhizospheric fluorescent Pseudomonas spp., RSB- rhizospheric Bacillus spp., EB- endophytic Bacillus spp.

Table.3 Over view of the results of bacterial inoculation on paddy growth in seedling bioassay

\begin{tabular}{|c|c|c|c|c|c|c|}
\hline \multirow[t]{3}{*}{ Isolates } & \multirow{3}{*}{$\begin{array}{l}\text { Total no. of } \\
\text { isolates } \\
\text { tested }\end{array}$} & \multicolumn{5}{|c|}{ Increase in total seedling length (\%) } \\
\hline & & $1-10$ & $11-20$ & $21-30$ & $31-40$ & $41-50$ \\
\hline & & \multicolumn{5}{|c|}{ Number of isolates } \\
\hline RSF & 21 & 5 & 1 & 4 & 1 & 1 \\
\hline RSB & 63 & 9 & 7 & 3 & 0 & 0 \\
\hline EB & 45 & 8 & 5 & 3 & 5 & 1 \\
\hline Total & 129 & 22 & 13 & 10 & 6 & 2 \\
\hline
\end{tabular}

RSF - rhizospheric fluorescent Pseudomonas spp., RSB- rhizospheric Bacillus spp., EB- endophytic Bacillus spp.

Table.4 Details of selected PGPR

\begin{tabular}{|c|c|c|c|c|}
\hline PGPR & $\begin{array}{l}\text { Coconut variety/ } \\
\text { Part of isolation }\end{array}$ & Place/State & $\begin{array}{l}\text { Medium } \\
\text { used for } \\
\text { isolation }\end{array}$ & $\begin{array}{l}\text { Assess } \\
\text { ment } \\
\text { score }\end{array}$ \\
\hline \multicolumn{5}{|c|}{ Bacillus spp. } \\
\hline ESB15 & \multirow{7}{*}{$\begin{array}{l}\text { WCT/Rhizosphere } \\
\text { WCT/Rhizosphere } \\
\text { TT/Rhizosphere } \\
\text { WCT/Rhizosphere } \\
\text { WCTxMYD/Rhizosphere } \\
\text { TT/Root } \\
\text { TT/Root }\end{array}$} & Ernakulam, Kerala & \multirow{2}{*}{$\begin{array}{l}\text { NA } \\
\text { NA }\end{array}$} & 13 \\
\hline KnSB6 & & Kunnamkai, Kasaragod, Kerala & & 12 \\
\hline TSB16 & & Tumkur, Karnataka & NA & 11 \\
\hline KiSB10 & & Kidu, Karnataka & NA & 12 \\
\hline RSB14 & & Ratnagiri, Maharashtra & NA & 16 \\
\hline TEB2 & & Tumkur, Karnataka & NA & 11 \\
\hline TEB4 & & Tumkur, Karnataka & NA & 11 \\
\hline HEB8 & \multirow{6}{*}{$\begin{array}{l}\text { WCT/Root } \\
\text { WCT/Root } \\
\text { WCT/Root } \\
\text { WCT/Root } \\
\text { WCT/Root } \\
\text { ECTxMYD/Root }\end{array}$} & HDMSCS, CPCRI, Kasaragod, Kerala & NA & 13 \\
\hline HEB10 & & HDMSCS, CPCRI, Kasaragod, Kerala & NA & 14 \\
\hline KiEB23 & & Kidu, Karnataka & NA & 11 \\
\hline KiEB25 & & Kidu, Karnataka & NA & 16 \\
\hline KiEB31 & & Kidu, Karnataka & NA & 11 \\
\hline PoEB5 & & Pollachi, Tamil Nadu & NA & \multirow[t]{2}{*}{17} \\
\hline \multicolumn{4}{|c|}{ Pseudomonas spp. } & \\
\hline \multirow{5}{*}{$\begin{array}{l}\text { ChSF180 } \\
\text { KnSF208 } \\
\text { ASF285 } \\
\text { PoSF314 } \\
\text { TSF7 } \\
\end{array}$} & \multirow{5}{*}{$\begin{array}{l}\text { WCT/Rhizosphere } \\
\text { WCT/Rhizosphere } \\
\text { ECT/Rhizosphere } \\
\text { ECTxMYD/Rhizosphere } \\
\text { TT/Rhizosphere }\end{array}$} & Chengannur, Alapuzha, Kerala & \multirow{2}{*}{$\begin{array}{l}\text { KBA } \\
\text { S2 }\end{array}$} & 12 \\
\hline & & Kunnamkai, Kasaragod, Kerala & & 12 \\
\hline & & Ambajipetta, Andhra Pradesh & S2 & 14 \\
\hline & & Pollachi, Tamil Nadu & KBA & 19 \\
\hline & & Tumkur, Karnataka & $\mathrm{KBA}$ & 17 \\
\hline RSF266 & WCTxMYD/Rhizosphere & Ratnagiri, Maharashtra & KBA & 12 \\
\hline KiSF27 & WCT/Rhizosphere & Kidu, Karnataka & S2 & 15 \\
\hline
\end{tabular}


Table.5 Plant growth promoting traits possessed by the selected PGPR

\begin{tabular}{|c|c|c|c|c|c|c|c|c|c|c|c|c|c|c|c|}
\hline \multirow[t]{4}{*}{$\begin{array}{r}\text { PGPR } \\
\end{array}$} & \multirow[b]{4}{*}{ Z } & \multirow{4}{*}{$\underbrace{\Xi}_{\mathscr{E}}$} & \multirow{4}{*}{ 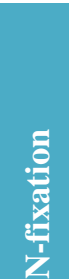 } & \multirow{4}{*}{ 冚 } & \multirow{4}{*}{$\frac{0}{\frac{0}{0}}$} & \multirow[b]{4}{*}{$\$$} & \multirow{4}{*}{ 己 } & \multirow{4}{*}{ 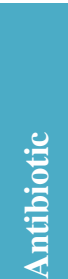 } & \multirow{4}{*}{ 卷 } & \multicolumn{2}{|c|}{ Antagonism * } & $\begin{array}{l}\text { Seedling } \\
\text { bioassay }\end{array}$ & \multicolumn{3}{|c|}{ Green house studies } \\
\hline & & & & & & & & & & & & \multirow{3}{*}{$\begin{array}{c}\% \\
\text { increase in } \\
\text { SL }\end{array}$} & \multicolumn{3}{|c|}{$\%$ increase in } \\
\hline & & & & & & & & & & & & & SL & FW & DW \\
\hline & & & & & & & & & & GA & $\mathbf{T P}$ & & & & \\
\hline Bacillus sp. ESB15 & + & - & - & M & $\mathrm{G}$ & - & $\mathrm{L}$ & - & 13 & 59 & 80 & 5 & 36 & - & 6 \\
\hline Bacillus sp. KnSB6 & + & - & - & $\mathrm{H}$ & - & - & $\mathrm{L}$ & - & 10 & 74 & 62 & 14 & 13 & 9 & - \\
\hline Bacillus sp. TSB16 & + & - & - & $\mathrm{H}$ & - & M & $\mathrm{L}$ & - & - & 57 & 56 & 11 & 43 & 65 & 163 \\
\hline Bacillus sp. KiSB10 & - & 10 & + & M & - & $\mathrm{H}$ & $\mathrm{L}$ & - & - & 71 & - & 7 & 29 & 50 & - \\
\hline Bacillus sp. RSB14 & - & 16 & + & M & 12 & - & $\mathrm{L}$ & - & 12 & 57 & 80 & 10 & 10 & 35 & 20 \\
\hline Bacillus sp. TEB2 & - & - & + & M & - & $\mathrm{L}$ & M & - & - & 57 & 64 & 20 & 21 & - & - \\
\hline Bacillus sp. TEB4 & - & - & + & $\mathrm{L}$ & - & M & $\mathrm{L}$ & - & - & 57 & 64 & 17 & 35 & - & 25 \\
\hline Bacillus sp. HEB8 & - & - & - & M & - & $\mathrm{H}$ & M & - & 11 & 74 & 56 & 30 & 30 & 21 & 25 \\
\hline Bacillus sp. HEB10 & - & - & - & $\mathrm{H}$ & - & M & M & - & 14 & 74 & 56 & 24 & 11 & - & - \\
\hline Bacillus sp. KiEB23 & - & 10 & + & $\mathrm{H}$ & G & - & - & - & - & 71 & 44 & 34 & 14 & 8 & 100 \\
\hline Bacillus sp. KiEB25 & - & 6 & + & $\mathrm{H}$ & - & - & $\mathrm{L}$ & - & 9 & 80 & 80 & 34 & 13 & 41 & 20 \\
\hline Bacillus sp. KiEB31 & - & 10 & + & $\mathrm{H}$ & - & - & $\mathrm{L}$ & - & - & 71 & 44 & 47 & 11 & - & 7 \\
\hline Bacillus sp. PoEB5 & + & 14 & + & M & 26 & - & $\mathrm{L}$ & - & - & 60 & 76 & 14 & 16 & 6 & - \\
\hline Pseudomonas sp. ChSF180 & - & 16 & - & $\mathrm{H}$ & 14 & $\mathrm{H}$ & $\mathrm{L}$ & - & - & - & 44 & 48 & 28 & 45 & 140 \\
\hline Pseudomonas sp. KnSF208 & - & 42 & - & $\mathrm{H}$ & 12 & $\mathrm{H}$ & $\mathrm{L}$ & - & - & - & - & 10 & 12 & 57 & 100 \\
\hline Pseudomonas sp. ASF285 & - & 16 & - & $\mathrm{L}$ & 12 & $\mathrm{~L}$ & $\mathrm{~L}$ & $\mathrm{~L}$ & - & 43 & 49 & 12 & 29 & 33 & 13 \\
\hline Pseudomonas sp. PoSF314 & - & 20 & - & M & 20 & $\mathrm{H}$ & $\mathrm{L}$ & $\mathrm{H}$ & - & 40 & 47 & 17 & 14 & 23 & 50 \\
\hline Pseudomonas sp. TSF7 & - & 16 & - & $\mathrm{H}$ & 16 & $\mathrm{H}$ & $\mathrm{L}$ & $\mathrm{H}$ & - & 43 & 56 & 21 & 33 & 14 & 50 \\
\hline Pseudomonas sp. RSF266 & - & 16 & - & M & 10 & $\mathrm{H}$ & $\mathrm{L}$ & $\mathrm{L}$ & - & - & 20 & 25 & 10 & 62 & 150 \\
\hline Pseudomonas sp. KiSF27 & - & 14 & + & $\mathbf{H}$ & 24 & $\mathbf{M}$ & $\mathbf{H}$ & - & - & - & - & 33 & 40 & 14 & - \\
\hline
\end{tabular}

HCN-Hydrogen cyanide production, PS- phosphate solubilization, N-fixation- capability to fix nitrogen, Ammonia-ammonification, Siderophore- siderophore production, IAA- IAA production, ACCd- ACC deaminase production, Antibiotic- antibiotic production, Chitinase- chitinase production, antagonism- $\%$ inhibition showed towards GA and TP on NA/KB, GA- antagonism against Ganoderma applanatum, TP- antagonism against Thielaviopsis paradoxa, +positive, - negative/no increase, H- high, M- medium, L-low, SL-seedling length, FW- fresh weight, DW- dry weight. 
Table.6 Identity of selected PGPR by $16 \mathrm{~S}$ rDNA sequencing and BLAST comparison

\begin{tabular}{|c|c|c|c|c|}
\hline $\begin{array}{l}\text { Sl. } \\
\text { No. }\end{array}$ & PGPR & Best classified BLAST hit & $\begin{array}{l}\text { Similarity } \\
(\%)\end{array}$ & $\begin{array}{c}\text { NCBI GenBank accession } \\
\text { numbers }\end{array}$ \\
\hline 1 & ChSF180 & Pseudomonas putida & 99 & KF381346 \\
\hline 2 & KnSF208 & P. putida & 99 & KF364491 \\
\hline 3 & ASF285 & P. monteilii & 99 & KF381348 \\
\hline 4 & PoSF314 & P. plecoglossicida & 99 & KF381347 \\
\hline 5 & TSF7 & P. monteilii & 99 & KF381345 \\
\hline 6 & RSF266 & P. plecoglossicida & 99 & KF381349 \\
\hline 7 & KiSF27 & P. plecoglossicida & 99 & KF381344 \\
\hline 8 & ESB15 & Bacillus cereus & 100 & KF381350 \\
\hline 9 & KnSB6 & B. cereus & 99 & KF381351 \\
\hline 10 & TSB 16 & B. megaterium & 99 & KF364492 \\
\hline 11 & KiSB10 & B. megaterium & 99 & KF381356 \\
\hline 12 & RSB14 & B. licheniformis & 99 & KF381343 \\
\hline 13 & TEB2 & B. megaterium & 100 & KF381342 \\
\hline 14 & TEB 4 & B. megaterium & 100 & KF381354 \\
\hline 15 & HEB 8 & B. cereus & 99 & KF381353 \\
\hline 16 & HEB 10 & B. cereus & 99 & KF381352 \\
\hline 17 & KiEB23 & Bacillus sp. & 98 & KF381357 \\
\hline 18 & KiEB25 & B. cereus & 100 & KF381358 \\
\hline 19 & KiEB31 & B. megaterium & 99 & KF381355 \\
\hline 20 & PoEB5 & B. subtilis & 99 & KF364490 \\
\hline
\end{tabular}

Table.S1 Effect of inoculation of rhizospheric fluorescent pseudomonads on paddy growth in seedling bioassay

\begin{tabular}{|c|c|c|c|}
\hline Treatment & $\begin{array}{l}\text { Root length* } \\
\text { (cm) }\end{array}$ & $\begin{array}{l}\text { Shoot length* } \\
(\mathrm{cm})\end{array}$ & $\begin{array}{l}\text { Total seedling } \\
\text { length* }(\mathrm{cm})\end{array}$ \\
\hline \multicolumn{4}{|l|}{ Experiment - I } \\
\hline Pseudomonas sp. HSF132 & \multirow{2}{*}{$\begin{array}{l}8.22 \mathrm{ab} \\
8.40 \mathrm{ab}\end{array}$} & $4.94 \mathrm{a}$ & $13.16 \mathrm{a}$ \\
\hline Pseudomonas sp. ESF153 & & $5.86 \mathrm{a}$ & $14.26 \mathrm{a}$ \\
\hline \multirow{2}{*}{$\begin{array}{l}\text { Pseudomonas sp. ESF168 } \\
\text { Pseudomonas sp. KnSF208 }\end{array}$} & \multirow{2}{*}{$\begin{array}{l}7.00 \mathrm{ab} \\
8.08 \mathrm{ab}\end{array}$} & $4.10 \mathrm{a}$ & $11.10 \mathrm{a}$ \\
\hline & & $4.86 \mathrm{a}$ & $12.94 \mathrm{a}$ \\
\hline Pseudomonas sp. RSF266 & $8.86 \mathrm{a}$ & $5.20 \mathrm{a}$ & $14.60 \mathrm{a}$ \\
\hline Pseudomonas sp. ASF285 & $8.54 \mathrm{ab}$ & $4.54 \mathrm{a}$ & 13.08 a \\
\hline Pseudomonas sp. PoSF315 & $4.50 \mathrm{~b}$ & $5.50 \mathrm{a}$ & $10.00 \mathrm{a}$ \\
\hline \multirow{2}{*}{$\begin{array}{c}\text { Pseudomonas sp. TSF7 } \\
\text { Control }\end{array}$} & \multirow{2}{*}{$\begin{array}{r}8.86 \mathrm{a} \\
6.90 \mathrm{ab}\end{array}$} & $5.36 \mathrm{a}$ & $14.22 \mathrm{a}$ \\
\hline & & $4.82 \mathrm{a}$ & $11.72 \mathrm{a}$ \\
\hline \multicolumn{4}{|l|}{ Experiment - II } \\
\hline \multirow{2}{*}{$\begin{array}{l}\text { Pseudomonas sp. ChSF180 } \\
\text { Pseudomonas sp. KiSF27 }\end{array}$} & \multirow{2}{*}{$\begin{array}{l}8.58 \mathrm{a} \\
8.65 \mathrm{a}\end{array}$} & $7.96 \mathrm{a}$ & $16.54 \mathrm{a}$ \\
\hline & & $6.20 \mathrm{ab}$ & $14.85 \mathrm{a}$ \\
\hline $\begin{array}{c}\text { Control } \\
\text { Experiment -III }\end{array}$ & $6.675 \mathrm{~b}$ & $4.525 \mathrm{~b}$ & $11.20 \mathrm{~b}$ \\
\hline \multirow{5}{*}{$\begin{array}{l}\text { Pseudomonas sp. RSF259 } \\
\text { Pseudomonas sp. KiSF13 } \\
\text { Pseudomonas sp. KiSF3 } \\
\text { Pseudomonas sp. KiSF16 } \\
\text { Pseudomonas sp. KiSF17 }\end{array}$} & \multirow{2}{*}{$\begin{array}{l}3.88 \mathrm{~b} \\
9.70 \mathrm{a}\end{array}$} & $5.82 \mathrm{bc}$ & $9.70 \mathrm{~b}$ \\
\hline & & $5.70 \mathrm{bc}$ & $15.40 \mathrm{a}$ \\
\hline & \multirow{2}{*}{$\begin{array}{l}8.14 \mathrm{a} \\
9.22 \mathrm{a}\end{array}$} & $6.80 \mathrm{ab}$ & $14.90 \mathrm{a}$ \\
\hline & & $7.36 \mathrm{ab}$ & $16.58 \mathrm{a}$ \\
\hline & $2.40 \mathrm{~b}$ & $6.32 \mathrm{abc}$ & $8.72 \mathrm{ab}$ \\
\hline \multirow{2}{*}{$\begin{array}{l}\text { Pseudomonas sp. KiSF21 } \\
\text { Pseudomonas sp. KiSF24 }\end{array}$} & $3.24 \mathrm{~b}$ & $5.20 \mathrm{c}$ & $8.44 \mathrm{~b}$ \\
\hline & $9.60 \mathrm{a}$ & $6.70 \mathrm{abc}$ & $16.30 \mathrm{a}$ \\
\hline Pseudomonas sp. KiSF28 & \multirow{2}{*}{$\begin{array}{l}3.54 \mathrm{~b} \\
8.10 \mathrm{a}\end{array}$} & $6.00 \mathrm{abc}$ & $9.54 \mathrm{~b}$ \\
\hline $\begin{array}{c}\text { Control } \\
\text { Experiment - IV }\end{array}$ & & $7.48 \mathrm{a}$ & 15.58 a \\
\hline \multirow{2}{*}{$\begin{array}{l}\text { Pseudomonas sp. RSF257 } \\
\text { Pseudomonas sp. PoSF313 }\end{array}$} & \multirow{2}{*}{$\begin{array}{l}9.50 \mathrm{a} \\
7.00 \mathrm{~b}\end{array}$} & $5.67 \mathrm{a}$ & $15.17 \mathrm{a}$ \\
\hline & & $4.68 \mathrm{a}$ & $11.68 \mathrm{~b}$ \\
\hline Pseudomonas sp. PoSF314 & $9.75 \mathrm{a}$ & $4.85 \mathrm{a}$ & $14.60 \mathrm{ab}$ \\
\hline Control & $7.33 \mathrm{~b}$ & $4.83 \mathrm{a}$ & $12.17 \mathrm{ab}$ \\
\hline
\end{tabular}

*Values are mean of 5 replications. Mean followed by the same letters in a column in an experiment are not significantly different from each other according to DMRT analysis at $\mathrm{P}=0.05$.

"Experiment were conducted in different batches with separate uninoculated control in each batch 
Table.S2 Effect of inoculation of rhizospheric Bacillus spp. on paddy growth in seedling bioassay

\begin{tabular}{|c|c|c|c|}
\hline Treatment & $\begin{array}{l}\text { Root length* } \\
(\mathrm{cm})\end{array}$ & $\begin{array}{l}\text { Shoot length* } \\
(\mathrm{cm})\end{array}$ & $\begin{array}{l}\text { Total seedling length* } \\
\text { (cm) }\end{array}$ \\
\hline Experiment $\mathrm{I}^{*}$ & & & \\
\hline Bacillus sp. KnSB22 & NG & NG & NG \\
\hline Bacillus sp. KnSB6 & $9.63 a b c$ & 6.26abcde & 15.89abed \\
\hline Bacillus sp. KnSB17 & $6.2 \mathrm{fg}$ & 6.4abcd & 12.6efg \\
\hline Bacillus sp. KnSB10 & $7.6 \mathrm{def}$ & 5.75cdefg & 13.35efg \\
\hline Bacillus sp. KnSB 19 & $6 f g$ & $6.05 \mathrm{bcdef}$ & $12.05 \mathrm{fg}$ \\
\hline Bacillus sp. KnSB7 & NG & NG & NG \\
\hline Bacillus sp. KnSB3 & $9.9 \mathrm{ab}$ & $7.46 \mathrm{a}$ & $17.36 \mathrm{ab}$ \\
\hline Bacillus sp. KnSB2 & $9.66 \mathrm{bcd}$ & 6.3abcde & $15.96 a b c$ \\
\hline Bacillus sp. HSB17 & $9.15 a b c$ & 5.3defg & $14.45 \mathrm{cdef}$ \\
\hline Bacillus sp. ChSB2 & $11.2 \mathrm{a}$ & $6.73 a b c$ & $17.93 \mathrm{a}$ \\
\hline Bacillus sp. ChSB4 & NG & NG & NG \\
\hline Bacillus sp. ChSB8 & $9.83 \mathrm{ab}$ & $6.8 \mathrm{abc}$ & $16.63 \mathrm{abc}$ \\
\hline Bacillus sp. ChSB9 & 8cde & $4.46 \mathrm{~g}$ & 12.46efg \\
\hline Bacillus sp. PSB13 & 6.6efg & $6.5 \mathrm{abcd}$ & 13.1efg \\
\hline Bacillus sp. ESB2 & $10 \mathrm{ab}$ & $7 a b c$ & 17ab \\
\hline Bacillus sp. ESB4 & 7.8def & $4.9 \mathrm{fg}$ & $12.7 \mathrm{efg}$ \\
\hline Bacillus sp. ESB8 & $5.6 \mathrm{~g}$ & $7.26 \mathrm{ab}$ & $12.86 \mathrm{efg}$ \\
\hline Bacillus sp. ESB13 & $7.63 \mathrm{def}$ & $7.46 \mathrm{ab}$ & 15.09bcde \\
\hline Bacillus sp. ESB14 & $5 \mathrm{~g}$ & 6.1abcdef & $11.1 \mathrm{~g}$ \\
\hline Bacillus sp. ESB15 & $7.5 \mathrm{def}$ & $7.1 \mathrm{abc}$ & 14.6cdef \\
\hline Control & $8.86 \mathrm{bcd}$ & $5.02 \mathrm{efg}$ & 13.88def \\
\hline Experiment II & & & \\
\hline Bacillus sp. KnSB13 & $5.30 \mathrm{~g}$ & $2.41 \mathrm{e}$ & $7.71 \mathrm{bc}$ \\
\hline Bacillus sp. KnSB15 & $5.05 \mathrm{~g}$ & $4.08 \mathrm{bc}$ & 9.13abc \\
\hline Bacillus sp. KnSB20 & $6.85 \mathrm{bcd}$ & $4.00 \mathrm{bcd}$ & $10.85 \mathrm{a}$ \\
\hline Bacillus sp. TSB13 & $6.16 \mathrm{e}$ & $3.73 \mathrm{bcd}$ & 9.89ab \\
\hline Bacillus sp. TSB16 & $6.49 \mathrm{cde}$ & $4.09 \mathrm{bc}$ & $10.58 \mathrm{a}$ \\
\hline Bacillus sp. CoSB1 & $5.53 \mathrm{fg}$ & $3.53 \mathrm{bcd}$ & $9.06 \mathrm{abc}$ \\
\hline Bacillus sp. CoSB5 & $6.32 \mathrm{de}$ & $3.75 \mathrm{bcd}$ & $10.07 \mathrm{a}$ \\
\hline Bacillus sp. PoSB7 & $5.0 \mathrm{~g}$ & $3.57 \mathrm{bcd}$ & 8.57abc \\
\hline Bacillus sp. RSB8 & $5.29 \mathrm{~g}$ & $3.30 \mathrm{~cd}$ & $8.59 \mathrm{abc}$ \\
\hline Bacillus sp. RSB14 & $7.32 \mathrm{~b}$ & $3.17 \mathrm{~d}$ & $10.49 \mathrm{a}$ \\
\hline Bacillus sp. ESB3 & $6.96 \mathrm{bc}$ & $3.67 \mathrm{bcd}$ & $10.63 \mathrm{a}$ \\
\hline Control & $5.97 \mathrm{ef}$ & $3.58 \mathrm{bcd}$ & $9.55 \mathrm{ab}$ \\
\hline Experiment III & & & \\
\hline Bacillus sp. CoSB3 & $11.86 \mathrm{~b}$ & $7.86 \mathrm{a}$ & $19.72 \mathrm{a}$ \\
\hline Bacillus sp. CoSB2 & $8.5 b$ & $6.8 \mathrm{abc}$ & $15.3 \mathrm{~b}$ \\
\hline Bacillus sp. CoSB4 & $10.14 \mathrm{~b}$ & $6.42 \mathrm{abc}$ & $16.56 \mathrm{~b}$ \\
\hline Bacillus sp. CoSB6 & $8.72 \mathrm{~b}$ & $6.76 a b c$ & $15.48 \mathrm{~b}$ \\
\hline Bacillus sp. CoSB7 & $6.16 \mathrm{~b}$ & $5.24 \mathrm{c}$ & $11.4 \mathrm{~b}$ \\
\hline Bacillus sp. CoSB8 & $9.5 \mathrm{~b}$ & $5.22 \mathrm{c}$ & $14.72 \mathrm{~b}$ \\
\hline Bacillus sp. CoSB10 & $9.94 \mathrm{~b}$ & $7.02 \mathrm{ab}$ & $16.96 \mathrm{~b}$ \\
\hline Bacillus sp. PoSB2 & $7.04 \mathrm{~b}$ & $5.65 \mathrm{bc}$ & $12.69 \mathrm{~b}$ \\
\hline Bacillus sp. PoSB8 & $8.26 \mathrm{~b}$ & $6.18 \mathrm{abc}$ & $14.44 \mathrm{~b}$ \\
\hline Bacillus sp. PoSB10 & $8.6 \mathrm{~b}$ & $6.5 \mathrm{abc}$ & $15.1 \mathrm{~b}$ \\
\hline Control & $12.42 \mathrm{~b}$ & $6.76 \mathrm{abc}$ & $19.18 b$ \\
\hline Experiment IV & & & \\
\hline Bacillus sp. ASB1 & $8.9 \mathrm{ab}$ & $5.68 \mathrm{abc}$ & 14.58ab \\
\hline Bacillus sp. ASB2 & $5.0 \mathrm{bc}$ & $5.26 \mathrm{abc}$ & $10.26 \mathrm{~b}$ \\
\hline Bacillus sp. ASB3 & $5.74 \mathrm{abc}$ & $5.84 \mathrm{abc}$ & 11.58ab \\
\hline Bacillus sp. ASB 13 & $8.76 \mathrm{ab}$ & $5.96 \mathrm{abc}$ & $14.72 \mathrm{ab}$ \\
\hline Bacillus sp. KiSB5 & $7.1 \mathrm{abc}$ & $6.06 \mathrm{abc}$ & $13.16 \mathrm{ab}$ \\
\hline Bacillus sp. KiSB9 & $9.15 \mathrm{ab}$ & $4.81 \mathrm{c}$ & $13.96 \mathrm{ab}$ \\
\hline Bacillus sp. KiSB10 & $8.6 \mathrm{abc}$ & $6.4 \mathrm{a}$ & $15.0 \mathrm{ab}$ \\
\hline Bacillus sp. KiSB21 & $5.12 b c$ & $6.28 \mathrm{abc}$ & $11.4 \mathrm{ab}$ \\
\hline Bacillus sp. KiSB22 & $6.54 a b c$ & $5.348 \mathrm{abc}$ & 11.888 ab \\
\hline Bacillus sp. KiSB24 & $7.34 \mathrm{abc}$ & $6.44 a$ & $13.78 \mathrm{ab}$ \\
\hline Bacillus sp. KiSB27 & $6.46 a b c$ & $5.74 a b c$ & $12.2 \mathrm{ab}$ \\
\hline Bacillus sp. KiSB34 & $5.628 \mathrm{abc}$ & $5.656 \mathrm{abc}$ & $11.284 \mathrm{ab}$ \\
\hline Bacillus sp. TSB2 & $8.57 \mathrm{ab}$ & $6.012 \mathrm{abc}$ & $14.582 \mathrm{ab}$ \\
\hline Bacillus sp. TSB3 & $5.44 a b c$ & $5.436 \mathrm{abc}$ & $10.876 \mathrm{~b}$ \\
\hline Bacillus sp. TSB8 & $6.04 a b c$ & $6.04 a b c$ & $12.08 \mathrm{ab}$ \\
\hline Bacillus sp. TSB21 & $5.2 \mathrm{bc}$ & $6.02 \mathrm{abc}$ & $11.22 \mathrm{ab}$ \\
\hline Bacillus sp. RSB3 & $4.064 \mathrm{c}$ & $6.32 \mathrm{ab}$ & $10.384 b$ \\
\hline Bacillus sp. RSB4 & $5.5 \mathrm{abc}$ & $5.86 \mathrm{abc}$ & $11.36 \mathrm{ab}$ \\
\hline Bacillus sp. RSB15 & 8.64ab & $4.884 \mathrm{bc}$ & $13.524 \mathrm{ab}$ \\
\hline Bacillus sp. RSB20 & $6.84 \mathrm{abc}$ & $5.4 \mathrm{abc}$ & $12.24 \mathrm{ab}$ \\
\hline Bacillus sp. RSB21 & $5.4 \mathrm{bc}$ & $5.8 \mathrm{abc}$ & $11.2 \mathrm{ab}$ \\
\hline Bacillus sp. RSB22 & $9.74 a$ & $6.0 \mathrm{abc}$ & $15.74 \mathrm{a}$ \\
\hline Control & $8.2 \mathrm{abc}$ & $5.802 \mathrm{abc}$ & $14.002 \mathrm{ab}$ \\
\hline
\end{tabular}

*Values are mean of 5 replications. Mean followed by the same letters in a column in an experiment are not significantly different from each other according to DMRT analysis at $\mathrm{P}=0.05$.

NG- No germination of paddy seeds

"Experiment were conducted in different batches with separate uninoculated control in each batch 
Table.S3 Effect of inoculation of endophytic Bacillus spp. on paddy growth in seedling bioassay

\begin{tabular}{|c|c|c|c|}
\hline Treatment & $\begin{array}{l}\text { Root } \\
\text { length* } \\
(\mathrm{cm})\end{array}$ & $\begin{array}{l}\text { Shoot } \\
\text { length* } \\
\text { (cm) }\end{array}$ & $\begin{array}{l}\text { Seedling } \\
\text { length* } \\
\text { (cm) }\end{array}$ \\
\hline \multicolumn{4}{|l|}{ Experiment $I^{\#}$} \\
\hline Bacillus sp. KiEB3 & $10.6 \mathrm{abc}$ & $6.44 a b c$ & $17.04 a b$ \\
\hline Bacillus sp. KiEB9 & 9.9abcd & $7.34 \mathrm{a}$ & $17.24 \mathrm{ab}$ \\
\hline Bacillus sp. KiEB13 & $9.0 \mathrm{bcde}$ & $5.42 \mathrm{~cd}$ & $14.42 \mathrm{~cd}$ \\
\hline Bacillus sp. KiEB14 & $9.2 \mathrm{bcde}$ & $6.96 \mathrm{ab}$ & 16.16abc \\
\hline Bacillus sp. KiEB15 & $9.94 \mathrm{abcd}$ & $6.92 \mathrm{ab}$ & 16.86ab \\
\hline Bacillus sp. KiEB16 & $8.22 \mathrm{cdef}$ & $5.62 \mathrm{bcd}$ & $13.84 \mathrm{bcd}$ \\
\hline Bacillus sp. KiEB19 & $5.12 f$ & $5.24 \mathrm{~cd}$ & $10.36 d$ \\
\hline Bacillus sp. KiEB20 & $7.36 \mathrm{cdef}$ & $6.26 \mathrm{abcd}$ & $13.62 \mathrm{bcd}$ \\
\hline Bacillus sp. KiEB23 & $12.94 \mathrm{a}$ & $5.98 \mathrm{bcd}$ & $18.92 a$ \\
\hline Bacillus sp. KiEB25 & $10.5 \mathrm{abc}$ & $6.3 \mathrm{abc}$ & 16.8ab \\
\hline Bacillus sp. KiEB18 & $6.7 \mathrm{def}$ & 6.06abcd & $12.76 \mathrm{bcd}$ \\
\hline Bacillus sp. KiEB26 & $6.1 \mathrm{ef}$ & $5.94 \mathrm{bcd}$ & $12.04 \mathrm{~cd}$ \\
\hline Bacillus sp. KiEB27 & 7.0def & 6.44abc & $13.44 \mathrm{bcd}$ \\
\hline Bacillus sp. KiEB29 & $6.98 \mathrm{def}$ & $6.46 a b c$ & $13.44 \mathrm{bcd}$ \\
\hline Bacillus sp. KiEB31 & $12.28 \mathrm{ab}$ & 6.26abcd & $18.54 a$ \\
\hline Control & $7.64 \mathrm{cdef}$ & $4.94 d$ & $12.58 b c d$ \\
\hline \multicolumn{4}{|l|}{ Experiment II } \\
\hline Bacillus sp. HEB7 & $6.8 \mathrm{bc}$ & $5.92 \mathrm{a}$ & $12.72 \mathrm{abc}$ \\
\hline Bacillus sp. HEB10 & $8.7 \mathrm{ab}$ & $5.76 \mathrm{a}$ & 14.46ab \\
\hline Bacillus sp. HEB6 & $6.68 b c$ & $4.5 \mathrm{a}$ & 11.18bed \\
\hline Bacillus sp. HEB1 & $5.3 \mathrm{~cd}$ & $4.6 \mathrm{a}$ & $9.9 \mathrm{~cd}$ \\
\hline Bacillus sp. HEB2 & $5.4 \mathrm{~cd}$ & $4.32 \mathrm{a}$ & $9.72 \mathrm{~cd}$ \\
\hline Bacillus sp. HEB16 & 7.64abc & $4.44 a$ & 12.08abc \\
\hline Bacillus sp. HEB8 & $9.86 \mathrm{a}$ & $5.3 \mathrm{a}$ & $15.16 \mathrm{a}$ \\
\hline Bacillus sp. TEB2 & $9.04 \mathrm{ab}$ & $4.98 \mathrm{a}$ & 14.02ab \\
\hline Bacillus sp. TEB4 & $8.6 \mathrm{ab}$ & $5.06 \mathrm{a}$ & 13.66ab \\
\hline Bacillus sp. TEB11 & $6.96 \mathrm{bc}$ & $4.8 \mathrm{a}$ & 11.96abc \\
\hline Bacillus sp. TEB20 & $3.2 \mathrm{~d}$ & $4.5 \mathrm{a}$ & $7.7 d$ \\
\hline Bacillus sp. TEB22 & 7.14abc & $4.52 \mathrm{a}$ & $11.66 a b c$ \\
\hline Control & $6.9 \mathrm{bc}$ & $4.82 \mathrm{a}$ & 11.7abc \\
\hline \multicolumn{4}{|l|}{ Experiment III } \\
\hline$\overline{\text { Bacillus sp. AEB5 }}$ & $14.44 \mathrm{a}$ & $6.68 \mathrm{ab}$ & $21.12 \mathrm{a}$ \\
\hline Bacillus sp. AEB10 & $11.6 \mathrm{abc}$ & $6.68 \mathrm{ab}$ & 17.84abc \\
\hline Bacillus sp. AEB11 & 10.68abcd & $5.16 \mathrm{ab}$ & 15.84bcd \\
\hline Bacillus sp. CoEB1 & $10.98 \mathrm{abc}$ & $6.88 \mathrm{a}$ & 17.86abc \\
\hline Bacillus sp. CoEB2 & $9.36 \mathrm{abcd}$ & $5.9 \mathrm{ab}$ & 15.26bcd \\
\hline Bacillus sp. CoEB3 & 12.42ab & $6.76 \mathrm{ab}$ & 19.18ab \\
\hline Bacillus sp. CoEB4 & $8.86 \mathrm{bcd}$ & $6.08 \mathrm{ab}$ & $14.94 \mathrm{bcd}$ \\
\hline Bacillus sp. CoEB5 & $9.52 \mathrm{abcd}$ & $6.54 \mathrm{ab}$ & 16.06abed \\
\hline Bacillus sp. CoEB6 & $8.48 \mathrm{bcd}$ & $4.98 \mathrm{~b}$ & $13.46 \mathrm{~cd}$ \\
\hline Bacillus sp. CoEB7 & $7.36 \mathrm{~cd}$ & $5.8 \mathrm{ab}$ & $13.16 \mathrm{~cd}$ \\
\hline Bacillus sp. CoEB8 & $6.12 \mathrm{~d}$ & $5.76 \mathrm{ab}$ & $11.88 \mathrm{~d}$ \\
\hline Bacillus sp. PoEB2 & $10.28 \mathrm{abcd}$ & $6.84 a$ & 17.12abc \\
\hline Bacillus sp. PoEB3 & $12.42 \mathrm{ab}$ & 6.76 & 19.18 \\
\hline Bacillus sp. PoEB4 & $12.68 \mathrm{ab}$ & $6.48 \mathrm{ab}$ & 19.16ab \\
\hline Control & $12.42 \mathrm{ab}$ & $6.76 \mathrm{ab}$ & 19.18ab \\
\hline \multicolumn{4}{|l|}{ Experiment IV } \\
\hline$\overline{\text { Bacillus sp. AEB3 }}$ & $5.71 \mathrm{~d}$ & $3.21 \mathrm{e}$ & $8.92 \mathrm{e}$ \\
\hline Bacillus sp. TEB18 & $6.53 b$ & $4.21 \mathrm{~b}$ & $10.74 b$ \\
\hline Bacillus sp. REB1 & $5.10 \mathrm{e}$ & $4.25 \mathrm{a}$ & 9.35d \\
\hline Bacillus sp. PoEB5 & $7.14 \mathrm{a}$ & $3.70 \mathrm{c}$ & $10.84 a$ \\
\hline Control & $5.97 \mathrm{c}$ & 3.58d & $9.55 \mathrm{c}$ \\
\hline
\end{tabular}

*Values are mean of 5 replications. Mean followed by the same letters in a column in an experiment are not significantly different from each other according to DMRT analysis at $\mathrm{P}=0.05$.

\#Experiment were conducted in different batches with separate uninoculated control in each batch 
Table.S4 Effect of inoculation of rhizospheric fluorescent pseudomonads on growth parameters of paddy seedlings in green house assay

\begin{tabular}{|c|c|c|c|c|c|c|c|}
\hline \multirow[t]{2}{*}{ Treatment } & \multirow{2}{*}{$\begin{array}{c}\text { Root } \\
\text { length* } \\
(\mathrm{cm})\end{array}$} & \multirow{2}{*}{$\begin{array}{l}\text { Shoot } \\
\text { length* } \\
\text { (cm) }\end{array}$} & \multirow{2}{*}{$\begin{array}{l}\text { Total } \\
\text { seedling } \\
\text { length* } \\
(\mathrm{cm}) \\
\end{array}$} & \multicolumn{3}{|c|}{$\%$ increase in } & \multirow{2}{*}{$\begin{array}{l}\log \\
\text { cfu/g } \\
\text { fresh } \\
\text { soil* }\end{array}$} \\
\hline & & & & TSL & FW & DW & \\
\hline Experiment - I & & & & & & & \\
\hline $\begin{array}{l}\text { Pseudomonas sp. } \\
\text { RSF257 }\end{array}$ & $13.67 \mathrm{~b}$ & $26.66 \mathrm{a}$ & $40.32 \mathrm{~b}$ & 4 & 62 & 113 & $5.52 \mathrm{a}$ \\
\hline $\begin{array}{l}\text { Pseudomonas sp. } \\
\text { KnSF208 }\end{array}$ & $17.22 \mathrm{ab}$ & $26.34 \mathrm{a}$ & $43.57 \mathrm{~b}$ & 12 & 57 & 100 & $5.50 \mathrm{a}$ \\
\hline $\begin{array}{l}\text { Pseudomonas sp. } \\
\text { RSF266 }\end{array}$ & $17.56 \mathrm{ab}$ & $25.24 \mathrm{a}$ & $42.80 \mathrm{~b}$ & 10 & 62 & 150 & $5.50 \mathrm{a}$ \\
\hline $\begin{array}{l}\text { Pseudomonas sp. } \\
\text { ASF285 }\end{array}$ & $21.14 \mathrm{a}$ & $29.09 \mathrm{a}$ & $50.23 \mathrm{a}$ & 29 & 33 & 13 & $5.50 \mathrm{a}$ \\
\hline $\begin{array}{l}\text { Control } \\
\text { Experiment - II }\end{array}$ & $15.58 \mathrm{~b}$ & $23.27 \mathrm{a}$ & $38.85 \mathrm{~b}$ & & & & $2 b$ \\
\hline $\begin{array}{l}\text { Pseudomonas sp. } \\
\text { ChSF180 }\end{array}$ & $9.76 \mathrm{a}$ & $18.82 \mathrm{ab}$ & $28.58 b$ & 25 & 45 & 140 & $5.79 a$ \\
\hline $\begin{array}{l}\text { Pseudomonas sp. } \\
\text { PoSF314 }\end{array}$ & $7.00 \mathrm{~b}$ & $18.60 \mathrm{ab}$ & $25.60 \mathrm{bc}$ & 12 & 23 & 50 & $5.65 a$ \\
\hline $\begin{array}{l}\text { Pseudomonas sp. } \\
\text { KiSF16 }\end{array}$ & $6.00 \mathrm{~b}$ & $14.00 \mathrm{c}$ & $20.00 \mathrm{~d}$ & - & - & 100 & $4.97 a$ \\
\hline $\begin{array}{l}\text { Pseudomonas sp. } \\
\text { KiSF27 }\end{array}$ & $10.45 \mathrm{a}$ & $20.83 \mathrm{a}$ & $31.28 \mathrm{a}$ & 36 & 14 & - & $5.92 \mathrm{a}$ \\
\hline $\begin{array}{l}\text { Pseudomonas sp. } \\
\text { TSF7 }\end{array}$ & $8.43 \mathrm{ab}$ & $18.50 \mathrm{ab}$ & $26.93 \mathrm{bc}$ & 17 & 14 & 50 & $5.79 a$ \\
\hline $\begin{array}{l}\text { Control } \\
\text { Experiment - III }\end{array}$ & $7.05 \mathrm{~b}$ & $15.88 \mathrm{c}$ & $22.93 \mathrm{~d}$ & & & & $2 \mathbf{b}$ \\
\hline $\begin{array}{l}\text { Pseudomonas sp. } \\
\text { KiSF24 }\end{array}$ & $13.34 \mathrm{ab}$ & $22.86 \mathrm{~b}$ & $36.20 \mathrm{bc}$ & 4 & 55 & 200 & $5.47 a$ \\
\hline $\begin{array}{l}\text { Pseudomonas sp. } \\
\text { HSF132 }\end{array}$ & $15.22 \mathrm{a}$ & $22.46 \mathrm{~b}$ & $37.680 \mathrm{bc}$ & 8 & 27 & 100 & $5.47 a$ \\
\hline $\begin{array}{l}\text { Pseudomonas sp. } \\
\text { ESF153 }\end{array}$ & $15.30 \mathrm{a}$ & $24.04 \mathrm{~b}$ & $39.34 \mathrm{ab}$ & 13 & 27 & 100 & $4.47 a$ \\
\hline Control & $12.20 \mathrm{~b}$ & $22.70 \mathrm{~b}$ & $34.90 \mathrm{c}$ & & & & $2 b$ \\
\hline
\end{tabular}

*Values are mean of 10 replications. Mean followed by the same letters in a column in an experiment are not significantly different from each other according to Duncan's multiple range test at $\mathrm{P}=0.05$.

TSL- total seedling length, FW- fresh weight, DW- dry weight, cfu- colony forming unit.

- No increase

"Experiment were conducted in different batches with separate uninoculated control in each batch

${ }^{\dagger}$ Population of fluorescent Pseudomonas spp. on s2 medium 
Table.S5 Effect of inoculation of rhizospheric Bacillus spp. on growth parameters of paddy seedlings in green house assay

\begin{tabular}{|c|c|c|c|c|c|c|c|}
\hline \multirow[t]{2}{*}{ Treatment } & \multirow{2}{*}{$\begin{array}{l}\text { Root } \\
\text { length* } \\
(\mathrm{cm})\end{array}$} & \multirow{2}{*}{$\begin{array}{l}\text { Shoot } \\
\text { length* } \\
(\mathrm{cm})\end{array}$} & \multirow{2}{*}{$\begin{array}{l}\text { Total } \\
\text { seedling } \\
\text { length* } \\
(\mathrm{cm})\end{array}$} & \multicolumn{3}{|c|}{$\%$ increase in } & \multirow{2}{*}{$\begin{array}{l}{ }^{\dagger} \log \text { cfu/g fresh } \\
\text { soil* }\end{array}$} \\
\hline & & & & TSL & FW & DW & \\
\hline \multicolumn{8}{|l|}{ Experiment $I^{\#}$} \\
\hline Bacillus sp. CoSB10 & $14.46 \mathrm{a}$ & $21.8 \mathrm{ab}$ & $36.26 \mathrm{a}$ & 7 & 8 & 50 & $6.46 \mathrm{ab}$ \\
\hline Bacillus sp. CoSB5 & $14.1 \mathrm{a}$ & $21.08 \mathrm{ab}$ & $35.18 \mathrm{a}$ & 3 & 4 & 50 & $6.51 \mathrm{ab}$ \\
\hline Bacillus sp. CoSB3 & $13.8 \mathrm{a}$ & $22.14 \mathrm{ab}$ & $35.94 \mathrm{a}$ & 6 & 9 & 50 & $6.20 \mathrm{a}$ \\
\hline Bacillus sp. RSB22 & $15.82 \mathrm{a}$ & $21.34 \mathrm{ab}$ & $37.16 \mathrm{a}$ & 9 & 6 & 50 & 6.67ab \\
\hline Bacillus sp. RSB15 & $13.68 \mathrm{a}$ & $20.56 a b$ & $34.24 \mathrm{a}$ & 1 & 2 & 50 & $7.15 a$ \\
\hline Bacillus sp. RSB3 & $14.42 \mathrm{a}$ & $21.9 \mathrm{ab}$ & $36.32 \mathrm{a}$ & 7 & 5 & - & 7.00a \\
\hline Bacillus sp. RSB14 & $14.00 \mathrm{a}$ & $23.5 \mathrm{a}$ & $37.50 \mathrm{a}$ & 10 & 35 & 20 & $6.73 \mathrm{ab}$ \\
\hline $\begin{array}{l}\text { Control } \\
\quad \text { Experiment II }\end{array}$ & $13.82 \mathrm{a}$ & $20.22 b$ & $34.04 \mathrm{a}$ & & & & $5.30 \mathrm{~b}$ \\
\hline Bacillus sp. KiSB5 & $11 \mathrm{~b}$ & $23.15 \mathrm{~cd}$ & $34.15 b c$ & 14 & 50 & - & $6.83 \mathrm{a}$ \\
\hline Bacillus sp. KiSB9 & $12.5 \mathrm{a}$ & $26 a b$ & $38.5 \mathrm{a}$ & 28 & 50 & - & $6.65 \mathrm{a}$ \\
\hline Bacillus sp. KiSB10 & $13.25 \mathrm{a}$ & $25.5 b$ & $38.75 a$ & 29 & 50 & - & $6.54 \mathrm{a}$ \\
\hline Bacillus sp. KiSB21 & $10.75 b$ & $24.85 b c$ & $35.6 b$ & 18 & 50 & - & $6.44 a$ \\
\hline Bacillus sp. KiSB24 & $7.6 \mathrm{~d}$ & $27.4 \mathrm{a}$ & $35 b$ & 16 & 50 & - & $6.43 \mathrm{a}$ \\
\hline Bacillus sp. ASB1 & $10.25 b c$ & $23.25 \mathrm{~cd}$ & $33.5 \mathrm{bc}$ & 11 & 50 & - & $6.51 \mathrm{a}$ \\
\hline Bacillus sp. ASB13 & $10.75 b$ & $21.5 \mathrm{de}$ & $32.25 \mathrm{c}$ & 7 & 100 & - & $6.68 \mathrm{a}$ \\
\hline $\begin{array}{l}\text { Control } \\
\text { Experiment III }\end{array}$ & \multicolumn{2}{|c|}{ Experiment III } & $30.05 \mathrm{~d}$ & 12 & & & $5.47 \mathrm{~b}$ \\
\hline Bacillus sp. KnSB2 & $18.14 \mathrm{a}$ & $25.72 \mathrm{a}$ & $43.86 \mathrm{ab}$ & 3 & - & - & $6.46 \mathrm{a}$ \\
\hline Bacillus sp. KnSB3 & $16.76 \mathrm{a}$ & $23.62 \mathrm{ab}$ & $40.38 \mathrm{ab}$ & 6 & 9 & - & $6.50 \mathrm{a}$ \\
\hline Bacillus sp. KnSB6 & $16.76 \mathrm{a}$ & 24.74ab & $41.5 \mathrm{ab}$ & 13 & 9 & - & $6.68 \mathrm{a}$ \\
\hline Bacillus sp. KnSB10 & $19.4 \mathrm{a}$ & $24.96 a$ & $44.36 \mathrm{a}$ & 12 & 9 & - & $6.30 \mathrm{a}$ \\
\hline Bacillus sp. KnSB15 & $17.88 \mathrm{a}$ & $26.14 \mathrm{a}$ & $44.02 \mathrm{ab}$ & 1 & 27 & - & $6.32 \mathrm{a}$ \\
\hline Bacillus sp. KnSB17 & $16.4 \mathrm{a}$ & $23.3 \mathrm{ab}$ & 39.7ab & 13 & - & - & $6.41 \mathrm{a}$ \\
\hline Bacillus sp. KnSB19 & $18.66 \mathrm{a}$ & $25.74 \mathrm{ab}$ & $44.4 \mathrm{ab}$ & 9 & 45 & - & $6.76 \mathrm{a}$ \\
\hline Bacillus sp. KnSB20 & $18.74 \mathrm{a}$ & $24 a b$ & $42.74 \mathrm{ab}$ & 12 & 18 & - & $6.72 a$ \\
\hline $\begin{array}{l}\text { Control } \\
\quad \text { Experiment IV }\end{array}$ & \multicolumn{7}{|c|}{ Experiment IV } \\
\hline Bacillus sp. PSB13 & $18.0 \mathrm{~cd}$ & $27.0 \mathrm{a}$ & $45.0 \mathrm{ab}$ & 17 & - & - & $6.95 a$ \\
\hline Bacillus sp. HSB17 & $19.9 \mathrm{ab}$ & $26.18 \mathrm{a}$ & $46.18 \mathrm{a}$ & 20 & 5 & - & $6.91 \mathrm{a}$ \\
\hline Bacillus sp. ESB2 & $19.24 b c$ & $25.54 \mathrm{a}$ & $44.78 \mathrm{ab}$ & 17 & - & - & $6.67 \mathrm{a}$ \\
\hline Bacillus sp. ESB3 & $18.3 \mathrm{bcd}$ & $27.48 \mathrm{a}$ & $45.78 \mathrm{a}$ & 19 & 11 & - & $6.32 \mathrm{a}$ \\
\hline Bacillus sp. ESB8 & $16.7 \mathrm{~d}$ & $25.12 \mathrm{a}$ & $41.82 b$ & 9 & - & - & $6.71 \mathrm{a}$ \\
\hline Bacillus sp. ChSB8 & $16.74 d$ & $25.4 \mathrm{a}$ & $42.5 b$ & 11 & - & - & $6.86 a$ \\
\hline Control & $21.2 \mathrm{a}$ & $17 . .18 \mathrm{~b}$ & $38.38 \mathrm{c}$ & & & & $\mathbf{5 b}$ \\
\hline \multicolumn{8}{|l|}{ Experiment $\mathbf{V}$} \\
\hline Bacillus sp. ESB13 & $20.42 a$ & $25.7 \mathrm{a}$ & $46.14 \mathrm{a}$ & 48 & 12 & 40 & $6.75 \mathrm{a}$ \\
\hline Bacillus sp. ESB14 & $16.48 b c$ & $24.76 \mathrm{ab}$ & $41.24 \mathrm{ab}$ & 33 & - & 23 & $6.55 \mathrm{a}$ \\
\hline Bacillus sp. ESB15 & $17.4 \mathrm{abc}$ & 24.94ab & $42.34 \mathrm{ab}$ & 36 & - & 6 & $5.95 \mathrm{a}$ \\
\hline Bacillus sp. TSB2 & $17.64 \mathrm{abc}$ & $24.2 \mathrm{ab}$ & $41.84 \mathrm{ab}$ & 35 & 18 & 40 & $6.61 \mathrm{a}$ \\
\hline Bacillus sp. TSB8 & $20.6 \mathrm{a}$ & $23.96 \mathrm{ab}$ & $44.56 \mathrm{ab}$ & 43 & 35 & 75 & $6.77 \mathrm{a}$ \\
\hline Bacillus sp. TSB13 & $17.9 \mathrm{abc}$ & $22.22 b$ & $40.12 b$ & 29 & - & 6 & 5.84ab \\
\hline Bacillus sp. TSB16 & $19.2 \mathrm{ab}$ & $25.14 \mathrm{ab}$ & $44.34 \mathrm{ab}$ & 43 & 65 & 163 & $6.47 \mathrm{a}$ \\
\hline Control & $15.79 \mathrm{c}$ & $15.3 \mathrm{c}$ & $31.09 c$ & & & & $5 b$ \\
\hline
\end{tabular}

*Values are mean of 10 replications. Mean followed by the same letters in a column in an experiment are not significantly different from each other according to Duncan's multiple range test at $\mathrm{P}=0.05$.

TSL- total seedling length, FW- fresh weight, DW- dry weight, cfu- colony forming unit.

- No increase

"Experiment were conducted in different batches with separate uninoculated control in each batch

${ }^{\dagger}$ Population of Bacillus spp. on NA 
Table.S6 Effect of inoculation of endophytic Bacillus spp. on growth parameters of paddy seedlings in green house assay

\begin{tabular}{|c|c|c|c|c|c|c|c|}
\hline \multirow[t]{2}{*}{ Treatment } & \multirow{2}{*}{$\begin{array}{l}\text { Root } \\
\text { length* } \\
(\mathrm{cm})\end{array}$} & \multirow{2}{*}{$\begin{array}{l}\text { Shoot } \\
\text { length* } \\
\text { (cm) }\end{array}$} & \multirow{2}{*}{$\begin{array}{l}\text { Total } \\
\text { seedling } \\
\text { length* } \\
(\mathrm{cm})\end{array}$} & \multicolumn{3}{|c|}{$\%$ increase in } & \multirow{2}{*}{$\begin{array}{c}\log \text { cfu/g } \\
\text { fresh } \\
\text { soil* }\end{array}$} \\
\hline & & & & TSL & FW & DW & \\
\hline Experiment $I^{\#}$ & & & & & & & \\
\hline Bacillus sp. PoEB3 & $15.5 \mathrm{bc}$ & $19.63 a$ & $35.13 \mathrm{ab}$ & 7 & 47 & 20 & $6.41 \mathrm{a}$ \\
\hline Bacillus sp. PoEB5 & $19.02 \mathrm{a}$ & $19.28 \mathrm{a}$ & $38.3 \mathrm{a}$ & 16 & 6 & - & 7.00a \\
\hline Bacillus sp. TEB18 & $13.72 \mathrm{c}$ & $20.66 a$ & $34.38 \mathrm{ab}$ & 4 & 47 & 20 & $6.53 \mathrm{a}$ \\
\hline Bacillus sp. KiEB25 & $18.02 \mathrm{ab}$ & $19.34 \mathrm{a}$ & $37.36 \mathrm{a}$ & 13 & 41 & 20 & $6.79 a$ \\
\hline Control & $15.38 \mathrm{bc}$ & $17.6 \mathrm{a}$ & $32.98 b$ & & & & $5.30 \mathrm{~b}$ \\
\hline Experiment II & & & & & & & \\
\hline Bacillus sp. HEB8 & $14.82 \mathrm{ab}$ & $22.03 \mathrm{a}$ & $36.85 a$ & 30 & 21 & 25 & $6.98 \mathrm{a}$ \\
\hline Bacillus sp. HEB10 & $13.68 \mathrm{bc}$ & $17.58 \mathrm{~b}$ & $31.26 \mathrm{bc}$ & 11 & - & - & $6.98 \mathrm{a}$ \\
\hline Bacillus sp. TEB2 & $14.36 \mathrm{abc}$ & $19.85 \mathrm{ab}$ & $34.21 \mathrm{ab}$ & 21 & - & - & 7.04a \\
\hline Bacillus sp. TEB4 & $17.32 \mathrm{a}$ & $20.7 \mathrm{a}$ & $38.02 \mathrm{a}$ & 35 & - & 25 & $7.17 a$ \\
\hline Bacillus sp. TEB22 & $14.4 \mathrm{abc}$ & $20.54 a$ & $34.94 \mathrm{ab}$ & 24 & - & - & $7.72 a$ \\
\hline Control & $10.85 \mathrm{c}$ & $17.39 \mathrm{~b}$ & $28.24 \mathrm{c}$ & & & & $5.30 \mathrm{~b}$ \\
\hline Experiment III & & & & & & & \\
\hline Bacillus sp. KiEB3 & $12.26 \mathrm{a}$ & $21.52 \mathrm{a}$ & $33.78 \mathrm{a}$ & 8 & 42 & 75 & $6.79 \mathrm{a}$ \\
\hline Bacillus sp. KiEB9 & $10.75 a$ & $20.56 a$ & $31.31 \mathrm{a}$ & - & - & 50 & $6.83 a$ \\
\hline Bacillus sp. KiEB13 & $12.1 \mathrm{a}$ & $19.1 \mathrm{a}$ & $31.2 \mathrm{a}$ & - & - & 75 & $2.311754 c$ \\
\hline Bacillus sp. KiEB14 & $12.14 \mathrm{a}$ & $21.73 a$ & $33.87 \mathrm{a}$ & 8 & - & 50 & $7.029384 a$ \\
\hline Bacillus sp. KiEB15 & $13.72 \mathrm{a}$ & $19.36 \mathrm{a}$ & $33.08 \mathrm{a}$ & 6 & - & 100 & $6.908485 a$ \\
\hline Bacillus sp. KiEB16 & $14.73 a$ & $20.87 \mathrm{a}$ & $35.6 \mathrm{a}$ & 14 & - & 100 & $6.672098 \mathrm{a}$ \\
\hline $\begin{array}{l}\text { Control } \\
\text { Experiment IV }\end{array}$ & $11.2 \mathrm{a}$ & $20.08 \mathrm{a}$ & $31.28 \mathrm{a}$ & & & & $5.30103 b$ \\
\hline Bacillus sp. KiEB23 & $16.6 \mathrm{~b}$ & $24.75 a$ & $41.35 \mathrm{a}$ & 14 & 8 & 100 & 7.133539a \\
\hline Bacillus sp. KiEB31 & $18.66 \mathrm{a}$ & $21.76 b c$ & $40.42 \mathrm{a}$ & 11 & - & 7 & $6.908485 a$ \\
\hline Bacillus sp. AEB5 & $12.27 \mathrm{~d}$ & $20.37 \mathrm{c}$ & $32.64 d$ & - & 23 & 33 & $6.869232 a$ \\
\hline Bacillus sp. REB1 & $17.98 \mathrm{ab}$ & $20.02 c$ & $38 \mathrm{~b}$ & 5 & - & 7 & $6.755875 a$ \\
\hline Bacillus sp. CoEB1 & $14.12 \mathrm{c}$ & $21.58 \mathrm{bc}$ & $35.7 b c$ & - & 54 & 87 & $1.869232 c$ \\
\hline Bacillus sp. CoEB3 & $11.86 \mathrm{~d}$ & $21.72 b c$ & $33.58 \mathrm{~cd}$ & - & 15 & 33 & $6.851258 \mathrm{a}$ \\
\hline Bacillus sp. PoEB2 & $17.54 \mathrm{ab}$ & $23.3 \mathrm{ab}$ & $40.84 a$ & 13 & 8 & 60 & $6.963788 a$ \\
\hline Bacillus sp. PoEB4 & $11.68 \mathrm{~d}$ & $21.84 \mathrm{bc}$ & $33.52 \mathrm{~cd}$ & - & 61 & 140 & $7.012837 a$ \\
\hline Control & $14.87 \mathrm{c}$ & $21.27 \mathrm{bc}$ & $36.14 \mathrm{~b}$ & & & & $5.380211 b$ \\
\hline Experiment V & & & & & & & \\
\hline Bacillus sp. HEB7 & $13.58 \mathrm{a}$ & $21.2 \mathrm{a}$ & $34.78 \mathrm{a}$ & 2 & 5 & - & $6.70757 a$ \\
\hline Bacillus sp. HEB16 & $13.24 \mathrm{a}$ & $20.08 \mathrm{a}$ & $33.32 \mathrm{a}$ & - & - & 100 & $6.763428 a$ \\
\hline Control & $13.82 \mathrm{a}$ & $20.22 a$ & $34.04 a$ & & & & $0.69897 b$ \\
\hline
\end{tabular}

*Values are mean of 10 replications. Mean followed by the same letters in a column in an experiment are not significantly different from each other according to Duncan's multiple range test at $\mathrm{P}=0.05$.

TSL- total seedling length, FW- fresh weight, DW- dry weight, cfu- colony forming unit.

- No increase

${ }^{\#}$ Experiment were conducted in different batches with separate uninoculated control in each batch

${ }^{\dagger}$ Population of Bacillus spp. on NA 
Fig.1 Isolation of fluorescent Pseudomonas spp. and Bacillus spp. a) Map indicating locations from which coconut roots and rhizospheric samples were collected (sample collection sites are marked with coconut palm and respective district names are given in red colour), b) fluorescent Pseudomonas spp. isolated on S2 agar, fluoresced on exposure to UV light, c) Bacillus spp. isolated on nutrient agar after heat treatment.

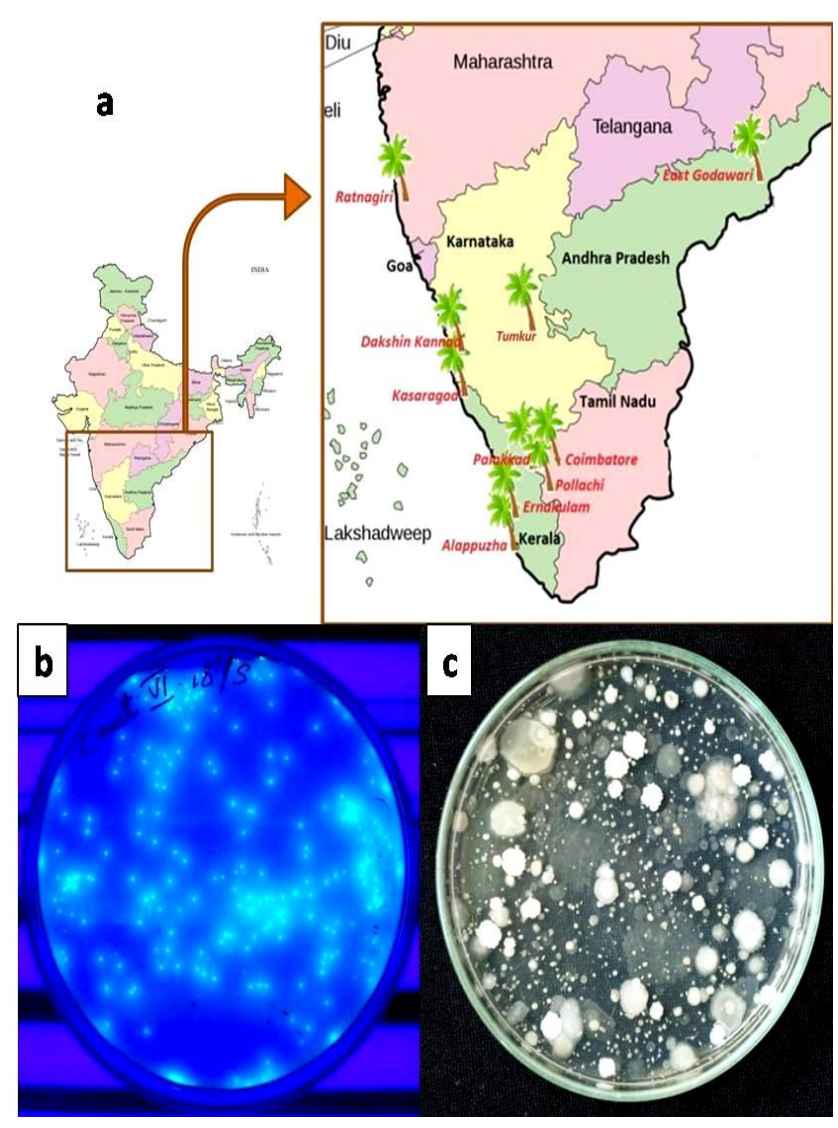


Fig.2 In vitro characterization of fluorescent pseudomonad isolates a) percentage of isolates showing plant growth promoting traits (HCN- hydrogen cyanide production, MPS- mineral phosphate solubilization, $\mathrm{N}$ - nitrogen fixation (presumptive), AMM- ammonia production, SIDsiderophore production, IAA- production of indole acetic acid, ACC- ACC deaminase activity, CHITIN- chitinase activity, ANTI- antibiotic production, G- antagonism against Ganoderma applanatum, TP- antagonism against Thielaviopsis paradoxa) b) characteristic pink colour developed by IAA producer, c) characteristic p-dissolution halo on Pikovskaya's agar, d) characteristic orange halos formed by siderophore producers on CAS agar, e) characteristic orange colour developed by HCN producer, f) characteristic clearing zone formed by chitinase producer, g) characteristic inhibition zone formed by antibiotic producer

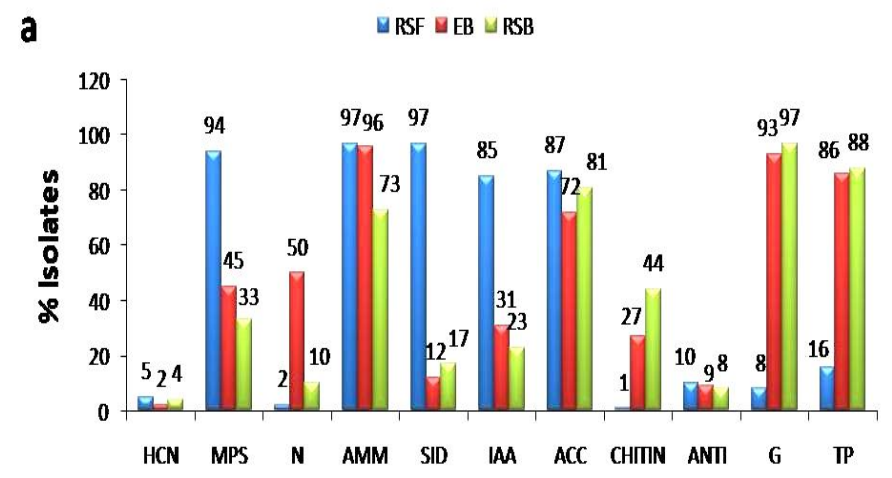

PGP traits

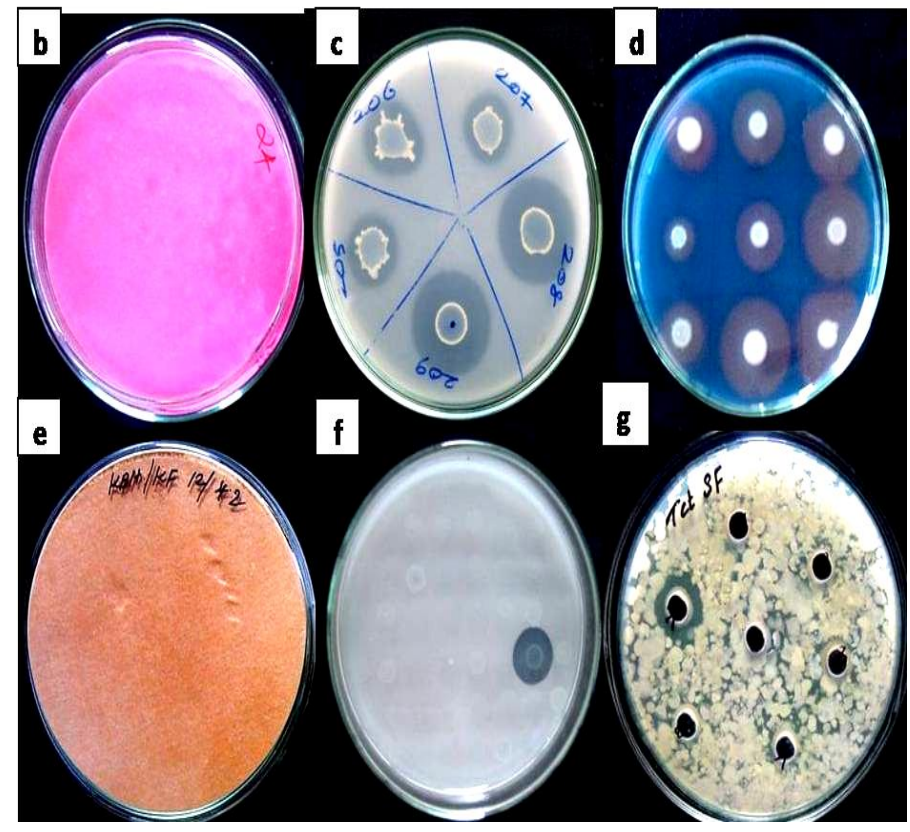


Fig.3 Antagonistic activity showed by isolates. a) Growth of Ganoderma applanatum on KBA, b) Growth of G. applanatum inhibited by RSF isolates on KBA, c) Growth of Thielaviopsis paradoxa on KBA, d) Growth of T. paradoxa inhibited by RSF on KBA, e) Growth of $G$. applanatum on NA, f) Growth of G. applanatum inhibited by Bacillus spp. on NA, g) Growth of T. paradoxa on NA, h) Growth of T. paradoxa inhibited by Bacillus spp. on NA.

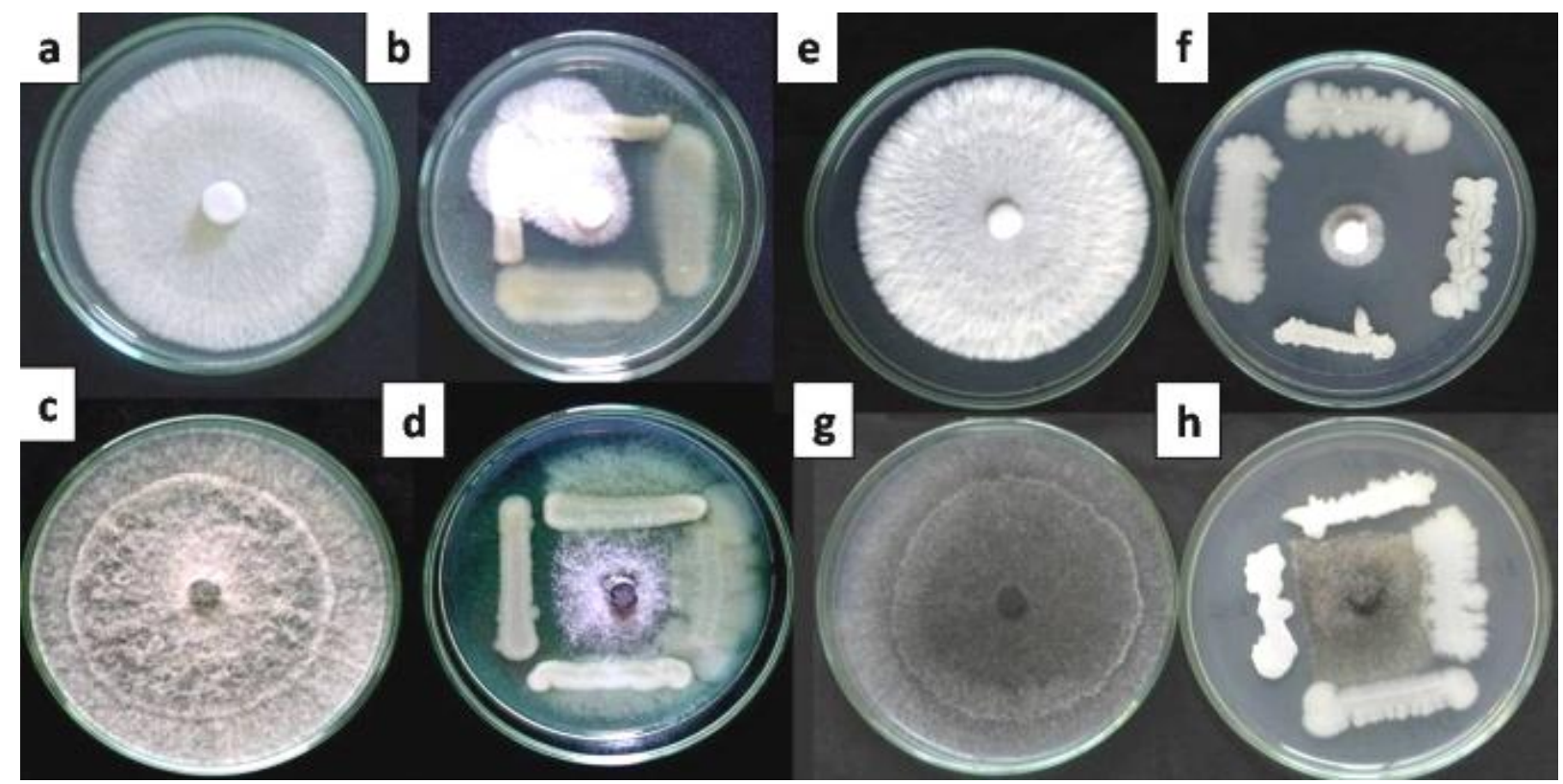

Fig.4 Effect of PGPR inoculations on (a) paddy seedlings under controlled light, temperature and humidity conditions, (b) paddy seedlings under green house conditions

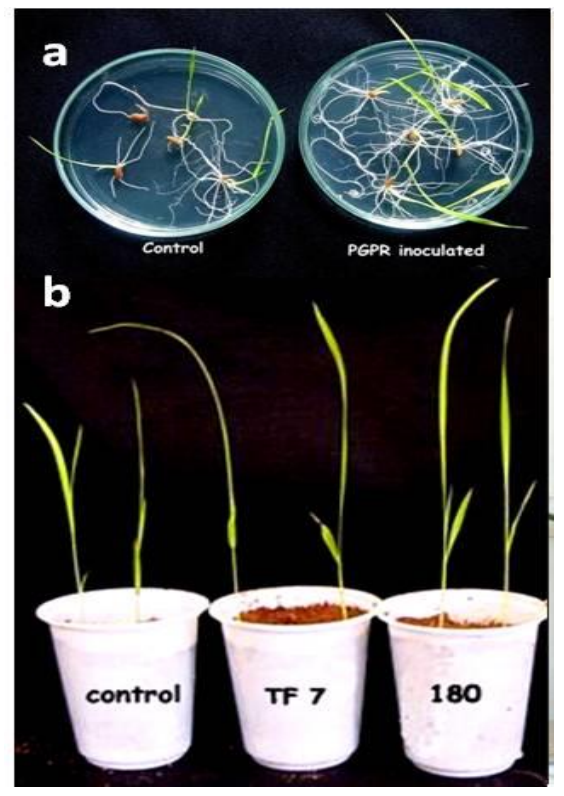


Sequence analysis of the 16S rRNA gene and BLAST sequence comparison confirmed the identity of isolates based on nucleotide homology and phylogenetic analysis as in the preliminary identification. The $16 \mathrm{~S}$ rDNA sequences were deposited in the NCBI Genbank and accession numbers obtained (Table 6).

Microbial enumeration and isolation studies in coconut palms growing in several parts of Kerala, Karnataka, Tamil Nadu, Andhra Pradesh and Maharashtra under different soil and ecological conditions clearly indicated that the fluorescent pseudomonads and Bacillus spp. community occurred in good numbers in the exo- or endo-rhizosphere of this perennial plantation crop thus confirming the presence of many types of plant beneficial microorganisms in coconut (George et al., 2011, 2012a, b). Fluorescent pseudomonads and Bacillus spp. are two important groups of PGPR already known for their plant growth promotion properties (Santoro et al., 2016; Govindasamy et al., 2010). We employed different approaches to determine the plant growth promoting potential of these fluorescent pseudomonads and Bacillus spp. isolated from coconut rhizosphere as well as the roots. This included in vitro testing of plant growth promotion traits, covering both direct and indirect modes of action (Gupta et al., 2000), plant growth promoting ability under controlled and uncontrolled greenhouse conditions.

All the isolated PGPR (483 numbers) from coconut exo- or endo-rhizosphere possessed at least one plant growth promoting traits in vitro. Among coconut endo or exorhizosphere bacteria, Bacillus spp. were found to be more dynamic in $\mathrm{N}$ - fixation ability (40\% of Bacillus spp.), chitin production (35\%) and antagonistic activity (95\% and $87 \%$ against $G$. applanatum and T. paradoxa, respectively), whereas Pseudomonas spp. were good in P-solubilization (94\% of RSF), siderophore production $(97 \%)$ and IAA production $(85 \%)$. There was a preponderance of ammonifying bacteria and antagonistic bacteria in the rhizosphere and roots of coconut. Ammonifying bacteria help in releasing ammonia $\left(\mathrm{NH}_{3}\right)$ from organic matters that are available for uptake by many plants. Seed inoculation of peanut with the ammonifying strain of Pseudomonas fluorescens PGPR1 increased the contents of nitrogen in soil, shoot and kernel of peanut (Dey et al., 2006). Rhizobacteria having biocontrol properties are considered as an alternative to chemical pesticides (Zahir et al., 2004). More than $87 \%$ of the Bacillus spp. were found antagonistic to G. applanatum and $T$. paradoxa, fungal pathogens of coconut. The results of our study confirmed the wide distribution of ACC deaminase activity in bacterial genera particularly Pseudomonas spp. (Klee et al., 1991) and Bacillus spp. (Ghosh et al., 2003) concurrent to previous studies from different crop soils. Although this study identified ACC deaminase activity of the isolates only qualitatively, this is the first study to report the presence of ACC degrading bacteria in the rhizosphere and roots of a perennial plantation crop like coconut. Another interesting result is that more than half of the bacterial isolates $(56 \%)$ associated with coconut exhibited phosphate solubilization activity as evidenced by the clearing zone around their colonies on Pikovskaya's agar. An increase in $P$ availability to plants through the use of phosphate solubilizing bacteria (PSB) had been reported under field conditions (Otieno et al., 2015). Bacteria with the ability to make atmospheric nitrogen in to available form play a critical role in the plant nutrition as nitrogen is one of the principal plant nutrients. $20 \%$ of the isolates showed growth on nitrogen-free medium indicating the ability to fix atmospheric nitrogen with the help of nitrogenase enzyme. Among 84 nitrogen 
fixers, 60 isolates were root endophytic Bacillus spp. Endophytic nitrogen fixing bacteria were reported from many crop plants like sugarcane, rice etc. (Muangthong et al., 2015; Ji et al., 2014). The production of phytohormones by PGPR is considered to be another important mechanism by which many rhizobacteria promote plant growth (Naveed et al., 2015). In this study, $45 \%$ of the total isolates, which included $86 \%$ of fluorescent Pseudomonas spp., showed the production of indole acetic acid (IAA) under in vitro conditions. The results confirmed the findings of Saharan and Nehra (2011) that Pseudomonas bacteria, especially $P$. fluorescens and $P$. putida, are the most important kinds of PGPR which produce auxins and promote the yield. The production of siderophores was found to be another most commonly observed plant growth promotion trait possessed by coconut PGPR. Siderophore production had been reported to be commonly associated with fluorescent Pseudomonas spp. (Bashan and de-Bashan, 2005). Fifteen percent of the Bacillus spp. from the rhizosphere and roots of coconut were also positive for siderophore production. Plants get benefit from this plant growth promotion trait in two ways: from the suppression of pathogens and from enhanced iron nutrition, resulting in increased plant growth (Bashan and de-Bashan, 2005; Gupta and Gopal, 2008). About one-fourth of the isolates exhibited chitinase activity among which Bacillus spp. were the predominant; about $35 \%$ of the Bacillus spp. exhibited chitinase production. Das et al., (2010) reported Bacillus sp., Serratia plymuthica, and Enterobacter agglomerans to be wellknown chitinolytic bacteria, which could be used as efficient biological control agents. The production of antibiotics is also considered as one of the most studied and powerful biocontrol mechanisms needed to be possessed by a PGPR for combating phytopathogens (Bashan and de-Bashan,
2005). The overall results of functional traits revealed that all the isolates showed strong tendencies for one or more of the PGPR traits tested and none were negative for all traits. All the isolates were assessed based on the performance in the in vitro plant growth promotion tests. An equal importance were given to all the plant growth promoting traits tested and maximum three points were possible for each parameter totaling to 33 points. The best performing isolates (129 nos.) were screened from the collection of 483 by assessing them according to their performance in plant growth promoting traits.

The approach of testing in vitro abilities has been proved to be an effective strategy to isolate PGPR; however, there are limitations. Some of the biochemical traits shown in vitro are inducible; i.e., they are expressed in certain conditions but not in others. Therefore, after the screening process, the potential PGPR needed to be tested in plants to ensure the same effect occurs in the plant too (Barriuso et al., 2008). Bacterial inoculation had been reported to have a positive influence on various plant-growth parameters, including root and shoot length, and dry biomass (Gupta et al., 1998; Nadeem et al., 2016). The outcome of our seedling bioassay under controlled conditions was that $41 \%$ (53 out of 129) isolates increased total seedling length with variable degree of stimulation, $14 \%$ showed inhibitory effect (non-significant in DMRT analysis) on plant growth and $44 \%$ remained as neutral compared to uninoculated control. The rhizosphere microorganisms, apart from being beneficial, are known to exert neutral and detrimental effects on plant growth and health (Gupta and Gopal, 1999).

Greenhouse studies further proved the efficiency of screened isolates for plant growth promotion in paddy seedlings. All isolates tested were found to be efficient in 
increasing either seedling length (up to $48 \%$ increase over uninoculated control), or fresh weight (up to $100 \%$ increase) or dry weight (up to $200 \%$ increase) of paddy seedlings. The strains that exhibited growth promotion of paddy seedlings in green house were very diverse in their traits. Therefore, growth enhancement by these organisms might involve more than one mechanism (Gupta et al., 2000). Pseudomonas monteilii PsF84 and Pseudomonas plecoglossicida PsF610, isolated from tannery sludge polluted soil, solubilized inorganic phosphorus and were capable of producing indole acetic acid (IAA) and siderophore. They increased the dry biomass of shoot, root, essential oil yield and chlorophyll of rose-scented geranium plants (Pelargonium graveolens cv. bourbon) over uninoculated control (Dharni et al., 2014). It had also been reported that the application of bacterial strains with multifaceted traits for plant growth promoting activity is more beneficial than with one plant growthpromoting trait (Indiragandhi et al., 2008). A total of 20 isolates (4\% of initial collection) were obtained as efficient PGPR with plant growth promoting abilities through our screening strategies. Some of the isolates selected from greenhouse assay possess direct plant growth promoting activity as well as biocontrol activity.

They inhibited the mycelial growth of Ganoderma applanatum and Thielaviopsis paradoxa in vitro. All the isolates improved paddy seedling growth (11-43\% over control) in controlled conditions as well as in uncontrolled greenhouse conditions. Their ability to retain their respective population in the rhizosphere of inoculated seedlings also added to their potency. These results are in agreement with other screening studies e.g., in Prunus root stocks, from a starting collection of several hundreds of strains, $20-25 \%$ exhibited certain degree of growth promotion activity, but in the final steps of selection, based on efficacy and consistency only 1-3\% were suitable for further use (Bonaterra et al., 2003). Out of 20 potent isolates, 15 exhibited antagonistic activity against both fungal pathogens of coconut, Ganoderma applanatum, and Thielaviopsis paradoxa. It is expected that plant growth promoting strains with high antagonistic potential will provide additional advantages to the seedlings (coconut) during exposure to pathogens. Identity of the finally selected PGPR was deduced using 16S rDNA sequence analysis with $99-100 \%$ similarity. All the identified isolates were reported as plant growth promoting rhizobacteria based on the plant growth promotion assays in greenhouse or field conditions (Dharni et al., 2014; Saharan and Nehra, 2011; Chakraborty et al., 2006; Govindasamy et al., 2010).

The most significant out come in this study is that strategic screening enabled to shortlist a total of 20 best PGPRs (7 Pseudomonas spp. and 13 Bacillus spp) with multifaceted traits from the preliminary collection of 483 isolates, based on their overall performance in in vitro characterization of plant growth promotion traits, seedling bioassay and green house trial. Utilization of these strains will enable to reduce the use of pesticides and fertilizers that are potential pollutants of the environment. The efficacy of the screened exo- or endo-rhizospheric bacterial isolates in improving the growth of coconut needs to be demonstrated and if further developed, could be recommended as efficient bioinoculants for growth and yield improvement in coconut.

\section{Acknowledgements}

We thank Indian Council of Agricultural Research, New Delhi for funding the Network Project on 'Application of Microorganisms in Agriculture and Allied Sectors', in which this work was carried out. Priya George is grateful for the award of Senior Research Fellowship. 


\section{References}

Amir, H.G., Shamsuddin, Z.H., Halimi, M.S., Marziah, M. and Ramlan, M.F. 2005. Enhancement in nutrient accumulation and growth of oil palm seedlings caused by PGPR under field nursery conditions. Communications in Soil Science and Plant Analysis. 36(15-16), 2059-2066.

Bakker, A.W. and Schippers, B. 1987. Microbial cyanide production in the rhizosphere in relation to potato yield reduction and Pseudomonas spp.mediated plant growth stimulation. Soil Biology and Biochemistry. 19, 451-457.

Barriuso, J., Beatriz, R., Solano, J.A., Lucas, Agustín, P.L., Ana, G., Francisco, J. and Gutiérrez, M. 2008. Ecology, Genetic Diversity and Screening Strategies of Plant Growth Promoting Rhizobacteria (PGPR). In: Ahmad, I., Pichtel, J., and Hayat, S. (Eds.), Plant-Bacteria Interactions. Strategies and Techniques to Promote Plant Growth. Wiley-VCH Verlag GmbH \& Co. KGaA, Weinheim, Germany, pp.1-17. doi:10.1002/ 9783527621989.ch1

Bashan, Y. and de-Bashan, L.E. 2005. Plant growth promoting bacteria. pp. 103115. In: Encyclopedia of soils in the environment, Elsevier, Oxford, U.K.Vol.1.

Bonaterra., Ruz, L., Badosa, E., Pinochet, J. and Montesinos, E. 2003. Growth promotion of Prunus rootstocks by root treatment with specific bacterial strains. Plant and Soil. 255, 555-569.

Brick, J.M., Bostock, R.M. and Silverstone, S.E. 1991. Rapid in situ assay for indole acetic acid production by bacteria immobilized on a nitrocellulose membrane. Applied Environment and Microbiology. 21, 613-618.
Cappuccino, J.C. and Sherman, N. 1992. In: Microbiology: A Laboratory Manual, New York, 125-179.

Chakraborty, U., Chakraborty, B. and Basnet, M. 2006. Plant growth promotion and induction of resistance in Camellia sinensis by Bacillus megaterium. Journal of basic microbiology. 46(3), 186-195.

Das, S.N., Sarma, P.V., Neeraja, C., Malati, N. and Podile, A.R. 2010. Members of Gamma proteobacteria and bacilli represent the culturable diversity of chitinolytic bacteria in chitin-enriched soils. World Journal of Microbiology and Biotechnology 26 (10), 1875-1881.

Dey, R., Pal, K.K., Bhatt, D.M. and Chauhan, S.M. 2006. Growth promotion and yield enhancement of peanut (Arachis hypogaea L.) by application of plant growth-promoting rhizobacteria. Microbiological Research 159(4), 371394.

Dharni, S., Srivastava, A.K., Samad, A. and Patra, D.D. 2014. Impact of plant growth promoting Pseudomonas monteilii PsF84 and Pseudomonas plecoglossicida PsF610 on metal uptake and production of secondary metabolite (monoterpenes) by rose-scented geranium (Pelargonium graveolenscv. bourbon) grown on tannery sludge amended soil. Chemosphere. 117, 433439.

Domenech, J., Ramos-Solano, B., Probanza, A., Lucas-Garcia, J., Colon, J.J. and Gutierrez-Manero, F.J. 2004. Bacillus spp. and Pisolithus tinctorius effects on Quercus ilex ssp. ballota: a study on tree growth, rhizosphere community structure and mycorrhizal infection. Forest Ecology Management. 194 (1), 293-303.

Dutta, J. and Thakur, D. 2017. Evaluation of multifarious plant growth promoting traits, antagonistic potential and 
phylogenetic affiliation of rhizobacteria associated with commercial tea plants grown in Darjeeling, India. PloS one. 3, 12(8): e0182302.

Fuhrmann, J. J. 1994. Isolation of soil microorganisms producing antibiotic compounds. pp. 379-405. In: Methods of Soil Analysis, Part 2, Microbiological and Biochemical Properties. Weaver, R.W. (Ed.) Soil Science Society of America, Madison, Wisconsin.

George, P., Gupta, A., Gopal, M., Chandra Mohanan, R., Thomas, L. and Thomas, G.V. 2012b. In vitro antagonism of rhizospheric fluorescent pseudomonads of coconut against Ganoderma applanatum and Thielaviopsis paradoxa, fungal pathogens of coconut. Journal of Plantation Crops. 40(2), 7581.

George, P., Gupta, A., Gopal, M., ChandraMohanan, R., Litty, T. and Thomas, G.V. 2011. Antagonistic activity of coconut rhizospheric and endophytic Bacillus spp. against Ganoderma applanatum and Thielaviopsis paradoxa. Journal of Plantation Crops. 39 (2), 278-284.

George, P., Gupta, A., Gopal, M., Thomas, L. and Thomas, G.V. 2013. Multifarious beneficial traits and plant growth promoting potential of Serratia marcescens KiSII and Enterobacter sp. RNF 267 isolated from the rhizosphere of coconut palms (Cocos nucifera L.). World Journal of Microbiology and Biotechnology. 29(1), 109-117.

George, P., Gupta, A., Gopal, M., Thomas, L. and Thomas, G.V. 2012a. Screening and in vitro evaluation of phosphate solubilizing bacteria from rhizosphere and roots of coconut palms (Cocos nucifera $\mathrm{L}$.) growing in different states of India. Journal of Plantation Crops. 40 (1), 61-64.
Ghosh, S., Penterman, J.N., Little, R.D., Chavez, R. and Glick, B.R. 2003. Three newly isolated plant growth-promoting bacilli facilitate the seedling growth of canola, Brassica campestris. Plant Physiology and Biochemistry. 41 (3), 277-281.

Gopal, M., Gupta, A. and Nair, R.V. 2005. Variations in hosting beneficial plantassociated microorganisms by root (wilt)-diseased and field-tolerant coconut palms of West Coast Tall variety. Current Science. 89 (11), 10-15.

Govindasamy, V., Senthilkumar, M., Magheshwaran, V., Kumar, U., Bose, P., Sharma, V. and Annapurna, K. 2010. Bacillus and Paenibacillus spp.: potential PGPR for sustainable agriculture In: Maheshwari, D.K. (Ed.), Plant growth and health promoting bacteria, Microbiology Monograph, 18, Springer, Berlin. pp 333-364.

Gupta, A. and Gopal, M. 1999. Plant growthinhibiting rhizosphere microorganisms: a review. Agricultural Reviews 20(2), $117-122$.

Gupta, A. and Gopal, M. 2008. Siderophore production by plant growth promoting rhizobacteria. Indian Journal of Agricultural Research. 42(2), 153-156.

Gupta, A., Gopal, M. and Tilak K.V.B.R. 2000. Mechanism of plant growth promotion by rhizobacteria. Indian Journal of Experimental Biology. 38(9), 856-862.

Gupta, A., Saxena, A.K., Gopal, M. and Tilak, K.V.B.R. 1998. Bacterization of green gram with rhizosphere bacteria for plant growth. Journal of Scientific and Industrial Research. 57, 726-731.

Gupta, A., Gopal, M. and Thomas, G.V. 2006. Bioaugmentation of Cocos nucifera $\mathrm{L}$. seedlings with the plant growth rhizobacteria, Bacillus coagulans and Brevibacillus brevis for growth 
promotion. In Proceedings of XXXVI Conference and Annual Meeting of European Society for New Methods in Agricultural Research, Iasi, Romania, Sept. 10-14, 2006.

Indiragandhi, P., Anandham, R., Madhaiyan, M. and Sa, T.M. 2008. Characterization of plant growth-promoting traits of bacteria isolated from larval guts of Diamondback Moth Plutella xylostella (Lepidoptera: Plutellidae). Current Microbiology. 56(4), 327-333.

Ji, S.H., Gururani, M.A. and Chun, S.C. 2014. Isolation and characterization of plant growth promoting endophytic diazotrophic bacteria from Korean rice cultivars. Microbiological research. 169(1):83-98.

Klee, H.J., Hayford, M.B., Kretzmer, K.A., Barry, G.F. and Kishore, G.M. 1991. Control of ethylene synthesis by expression of a bacterial enzyme in transgenic tomato plants. Plant cell 3, 1187-1193.

Lucy, M., Reed, E. and Glick, B.R. 2004. Application of free living plant growthpromoting rhizobacteria. Antonie Leeuwenhoek 86 (1), 1-25.

Mantelin, S. and Touraine, B. 2004. Plant growth-promoting bacteria and nitrate availability: impacts on root development and nitrate uptake. Journal of Experimental Botony. 55 (394), 27 34.

Mendes, R., Garbeva, P. and Raaijmakers, J.M. 2013. The rhizosphere microbiome: significance of plant beneficial, plant pathogenic, and human pathogenic microorganisms. FEMS microbiology reviews. 37(5), 634-663.

Muangthong, A., Youpensuk, S. and Rerkasem, B. 2015. Isolation and characterisation of endophytic nitrogen fixing bacteria in sugarcane. Tropical life sciences research. 26(1), 41.
Nadeem, S.M., Ahmad, M., Naveed, M., Imran, M., Zahir, Z.A. and Crowley, D.E. 2016. Relationship between in vitro characterization and comparative efficacy of plant growth-promoting rhizobacteria for improving cucumber salt tolerance. Archives of microbiology. 198(4), 379-387.

Naveed, M., Qureshi, M.A., Zahir, Z.A., Hussain, M.B., Sessitsch, A. and Mitter, B. 2015. L-Tryptophan-dependent biosynthesis of indole-3-acetic acid (IAA) improves plant growth and colonization of maize by Burkholderia phytofirmans PsJN. Annals of microbiology. 65(3), 1381-1389.

Otieno, N., Lally, R.D., Kiwanuka, S., Lloyd, A., Ryan, D., Germaine, K.J. and Dowling DN. 2015. Plant growth promotion induced by phosphate solubilizing endophytic Pseudomonas isolates. Frontiers in Microbiology. 6, 745.

Rajendran, L., Karthikeyan, G., Raguchander, T. and Samiyappan, R. 2007. In vitro evaluation of bacterial endophytes influence on Ganoderma lucidum (leys) karst. mycelial growth. J. Plant Protection Res. 47(4), 426-436.

Renwick, A., Campbell, R. and Coe, S. 1991. Assessment of in vivo screening systems for potential biocontrol agents of Gaeumannomyces graminis. Plant Pathol. 40, 524-532.

Saharan, B.S. and Nehra, V. 2011. Plant growth promoting rhizobacteria: a critical review. Life Sci. Med. Res. 21, $1-30$.

Santoro, M.V., Bogino, P.C., Nocelli, N., Del Rosario Cappellari, L., Giordano, W.F. and Banchio, E. 2016. Analysis of plant growth-promoting effects of fluorescent Pseudomonas strains isolated from Mentha piperita rhizosphere and effects of their volatile organic compounds on 
essential oil composition. Frontiers in microbiology. 7.

Schwyn, B. and Nielands, J.B. 1987. Universal chemical assay for the detection and determination of siderophores. Anal. Biochem. 160, 4756.

Thomas, L., Gupta, A., Gopal, M., George, P and Thomas, G.V. 2011. Efficacy of rhizospheric Bacillus spp. for growth promotion in Theobroma cacao $\mathrm{L}$. seedlings. Journal of Plantation Crops. 39(1), 19-25.
Vargas, L.K., Volpiano, C.G., Lisboa, B.B., Giongo, A., Beneduzi, A. and Passaglia, L.M. 2017. Potential of rhizobia as plant growth-promoting rhizobacteria. In Microbes for legume improvement, Springer, Cham. pp. 153-174.

Zahir, Z.A, Arshad M. and Frankenberger, W.T. 2004. Plant growth promoting rhizobacteria: applications and perspectives in agriculture. Adv. Agron. 81, 97-168.

\section{How to cite this article:}

Priya George, Alka Gupta, Murali Gopal, Litty Thomas and George V. Thomas. 2018. Systematic Screening Strategies for Identifying Elite Plant Growth Promoting Rhizobacteria for Coconut (Cocos nucifera L.). Int.J.Curr.Microbiol.App.Sci. 7(05): 1051-1074. doi: https://doi.org/10.20546/ijcmas.2018.705.130 